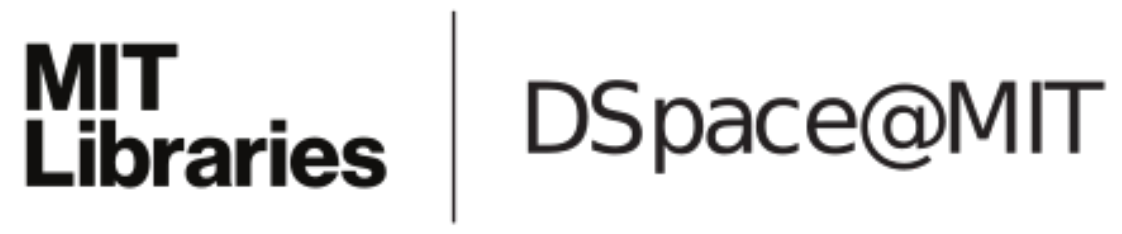

\author{
MIT Open Access Articles
}

Optimal Planning Quantities for Product Transition

The MIT Faculty has made this article openly available. Please share how this access benefits you. Your story matters.

Citation: Li, H., Graves, S. C. and Rosenfield, D. B. (2010), Optimal Planning Quantities for Product Transition. Production and Operations Management, 19: 142-155. doi: 10.1111/ j.1937-5956.2009.01091.x

As Published: http://dx.doi.org/10.1111/j.1937-5956.2009.01091.x

Publisher: Wiley Blackwell (Blackwell Publishing)

Persistent URL: http://hdl.handle.net/1721.1/69643

Version: Author's final manuscript: final author's manuscript post peer review, without publisher's formatting or copy editing

Terms of use: Creative Commons Attribution-Noncommercial-Share Alike 3.0 


\title{
Optimal Planning Quantities for Product Transition
}

\author{
November 2007, revised June 2008, April 2009
}

\author{
Hongmin Li \\ W.P. Carey School of Business, Arizona State University \\ Department of Supply Chain Management \\ P.O. Box 874706, Tempe, Arizona 85287-4706 \\ Telephone: 480-965-2232 \\ Fax: 480-965-8629 \\ E-mail:hongmin.li@asu.edu
}

Stephen C. Graves

Sloan School of Management, Massachusetts Institute of Technology

77 Massachusetts Avenue

Building E53-347

Cambridge, MA 02139-4307

Telephone: 617-253-6602

Fax: 617-258-7579

E-mail:sgraves@mit.edu

Donald B. Rosenfield

Sloan School of Management, Massachusetts Institute of Technology

77 Massachusetts Ave.

Building E40-319

Cambridge, MA 02139-4307

Telephone: 617-253-1064

Fax: 617-253-1462

E-mail:donrose@mit.edu 


\title{
Optimal Planning Quantities for Product Transition
}

\author{
Abstract \\ The replacement of an existing product with a new one presents many challenges. In particular, \\ uncertainties in a new product introduction often lead to extreme cases of demand and supply \\ mismatches. This paper addresses inventory planning decisions for product upgrades when there \\ is no replenishment opportunity during the transition period. We allow product substitution: \\ when a company runs out of the old product, a customer may be offered the new product as a \\ substitute. We show that the optimal substitution decision is a time-varying threshold policy and \\ establish the optimal planning policy. Further, we determine the optimal delay in a new product \\ introduction, given the initial inventory of the old product.
}

\section{Keywords: inventory planning; substitution; product transition; new product introduction}

Received November 2007; revisions received August 2008 and April 2009; accepted April 2009

\section{Introduction}

As product life cycles become shorter, managing product transition has become a major challenge for companies in fast clockspeed industries. We consider the problem of production and inventory planning when a product is replaced by its next generation counterpart. In particular, we study these decisions when neither product can be replenished during the transition period.

This research originates from collaborative work with a telecommunications equipment manufacturer. The company frequently updates its products due to technology change. Product lifecycles can range from 15 to 24 months and new products account for about one third of the company's annual revenue. For each product transition, operations managers need to make planning decisions that are complicated by several factors: the procurement and production lead-times 
can be quite long relative to the transition period; transition-period demand for both the new and old products is uncertain; and product substitution can occur when one product stocks out.

In this industry, the procurement lead-time for critical components can be 13 weeks or longer. The production lead time is usually 5 weeks. As a result, planning decisions need to be made 4 to 6 months prior to the product transition, which starts when the new product is released and ready to ship to customers. The transition ends when the new-product demand rate stabilizes and that of the old drops to a negligible level. This transition can take between a few weeks to a few months, during which the company sells both products. As the transition period is typically shorter than the replenishment lead time, any replenishment ordered during the transition will not arrive until after the transition is completed. The planning challenge is to assure enough supply for both products but not too much.

A further complication for the transition planning is the opportunity for product substitution during the transition: often when a shortage of the old product occurs, the new product can be used as a substitute. The product manager needs to decide when to offer substitution to customers and to understand how this substitution decision affects the initial planning quantities.

Additionally a new-product launch involves many sequential steps and depends on many factors including technology, production and supplies. As a result, a new product release schedule often slips. When this happens, the old-product inventory may run out, creating a supply gap.

As an example, our collaborating company was replacing a wireless access point product named Blofeld, with its next generation successor Blofeld2. The transition was driven in part by an end-of-life (EOL) notice from a component supplier for Blofeld. The company did not buy many old products on the EOL notice, hoping that Blofeld2 would cover the demand. Unfortunately, the Blofeld2 release was delayed, resulting in a supply gap. As it was impossible to re- 
plenish Blofeld, the company had to scramble to complete Blofeld2 to narrow the supply gap.

As another example, the old product, Sultan, was selling very well before the transition. Unwilling to risk any supply gap and miss revenues, the managers made large additional purchases of the old product as buffer inventory. This time, all work leading to the transition had gone better than planned and the new product, Sultan II, was completed on schedule. This created another dilemma as the company had a large inventory position in the old product, both on-hand and onorder. Releasing Sultan II as scheduled would likely result in a large excess of Sultan. The company decided to delay the new product introduction in order to mitigate the excess problem.

In this paper we address the planning challenge for a product transition, namely to balance the risk of shortage against the risk of excess. This problem has been identified by companies in various industries including Intel (Hopman 2005) and Lexmark (Saenz et al. 2005), but has not drawn much academic attention. More specifically we examine two decisions.

First, we address the inventory planning problem that determines the quantities of the old and the new products, accounting for uncertainties in demand and in the new-product release date. We develop a dynamic model for joint inventory decisions for two generations of products, with non-stationary and substitutable demand. The substitution decision is a dynamic decision that is contingent upon the inventory levels of both products and the time during a transition.

Second, we address a closely related problem. In the Sultan transition, the decision to postpone the transition was driven by the need to consume excess inventory and to avoid a large obsolescence cost. However, since the new product typically has better margins, such a delay can hurt the bottom line. Therefore, the decision of when to launch the new product is a tradeoff of these two conflicting objectives. In this paper, we determine whether and by how much to delay a new product release when a company is carrying a large inventory of the old product. 
We use the term "new product release date" and "transition start date" (TSD) interchangeably, as we assume that a product transition starts when the new product is released. The proofs of the results in this paper are provided in the Online Supplement at http:/www. poms.org/journal/supplements/.

\section{Literature Review}

Existing literature on single-product inventory planning problems abounds. One stream concerns the disposal of the old generation product - the last time buy decisions. Goyal and Giri (2001) provide a general review on this subject. Rosenfield $(1989,1992)$ presents a marginal analysis model that solves for the optimal number of units to keep for products that are slow-moving or obsolete when facing a one-time disposal opportunity. Jain and Silver (1994) develop a dynamic programming model to determine the ordering policy for a product with a random lifetime and stochastic but stationary demand. Krishnamoorthy and Varghese (1995), Kalpakam and Sapna (1994) and Ravichandran (1995) develop continuous inventory replenishment models for a product with random lifetime and stationary demand. Song and Zipkin (1996) examine an inventory control problem for a deteriorating demand situation; they model the state of the demand process as a Markov chain that alternates between low and high demand states. In contrast, we allow the expected demand rate to change continuously.

There are two streams of literature on inventory models for multiple products with substitutions. One stream focuses on static substitutions in a single-period or repeated newsvendor problem. Parlar and Goyal (1984), Pasternack and Drezner (1991), and Bassok et al. (1999) consider a single-period multiple-product inventory model with substitutions. Rao et al. (2004) study a similar problem with setup costs. Bitran and Dasu (1992) model a multi-period production planning problem with demand substitutions and both supply and demand uncertainty. Another 
stream of substitution literature couples the customers' choice model with the newsvendor problem to derive optimal order quantities. Smith and Agrawal (2000) consider a single-period multiitem stocking problem using demands modified by customers' substitution effects. Mahajan and van Ryzin (2001) analyze the same problem, but the customer's substitution choice depends upon the current inventory levels. Although this is similar to the substitution model in this paper, we look at substitution decisions that are initiated by the firm, instead of the customers.

Billington et al. (1998) provide a comprehensive framework for product "rollover" strategies. They discuss both primary strategies for decision-making before the rollover process starts and contingency strategies that help a company adapt to additional market and product information as it becomes available. In our paper, we address both primary and contingency strategies. In the inventory planning problem, we consider primary strategies, namely the initial inventory decisions and the release time for the new product. But for our analysis of these decisions, we incorporate a dynamic substitution decision, which is a contingency strategy.

Wilhelm and $\mathrm{Xu}(2002)$ examine a problem that is similar to ours. They consider a multiperiod production planning and pricing problem in a product upgrade, for which there are replenishment opportunities in each period. They assume that only one product is sold at any point of time, i.e., a "solo-product roll" as defined by Billington et al (1998). In contrast, we allow both generation products to be sold throughout the transition period (a "dual-product roll") and solve the planning problem when there is no additional replenishment opportunity.

Souza et al. (2004) study the optimal timing and production decisions for new product introductions in a duopoly situation under the "solo-product roll" assumption. They determine, for each time period, whether to introduce a new product with "incremental" or "substantial" improvements, or not to introduce at all. Savin and Terwiesch (2005) adopt a competitive diffusion 
model to study the optimal product launch time in a duopoly. They model the tradeoff between life-cycle sales of the product and production cost reduction caused by changes in the launch time. Wilson and Norton (1989) consider the optimal entry timing for a product line extension. The tradeoff is between the market growth stimulated by the new product and the sales cannibalization of the old product due to the product line extension. In contrast to the above papers, we focus on the operational tradeoffs (excess inventory versus improved margin from the new product) when determining the optimal date for a new product release.

\section{Problem Description}

We explore the following three problems regarding a product transition.

1) Given a deterministic transition start date (TSD), what are the optimal planning quantities for the old and new products? To determine the initial inventory positions, we examine a dynamic decision of whether to offer substitution to customers when the old product runs out.

2) Given a stochastic TSD, what are the optimal planning quantities for each product? We compare the optimal solutions with the case of a deterministic TSD to assess the impact of this uncertainty on the optimal planning quantities and on the total expected profit.

3) Given an initial scheduled TSD and the initial inventory position of the old product (on hand plus on order), should the firm delay the transition? If so, by how much?

We study the above problems for a finite time horizon of length $H$. We define time zero to be the time when the planning decisions need to be made. We define $\tau$ to be the date when the transition starts and we assume that the planning horizon $H$ extends beyond the end of the transition. $H$ can signify a fiscal target date such as the end of a month or a quarter, or simply a time for making the next replenishment decision. We define the transition period to be the time interval $[\tau, H]$. The lead-time to replenish either product is long, relative to the duration of the transition; 
thus we assume that there is only one order opportunity and it takes place at time zero.

We assume that the demands of the two products are independent Poisson processes throughout the horizon $[0, H]$. For the new product, the demand rate is zero prior to time $\tau$, is non-decreasing during the transition period and then stabilizes at a constant level. For the old product, the demand rate before the transition is constant, is non-increasing during the transition period and then falls to a negligible level after the transition. Thus, the company sells both products during the transition period $[\tau, H]$ as customers gradually switch over from the old to the new product. Figure 1 illustrates a typical pattern for these demand rates.

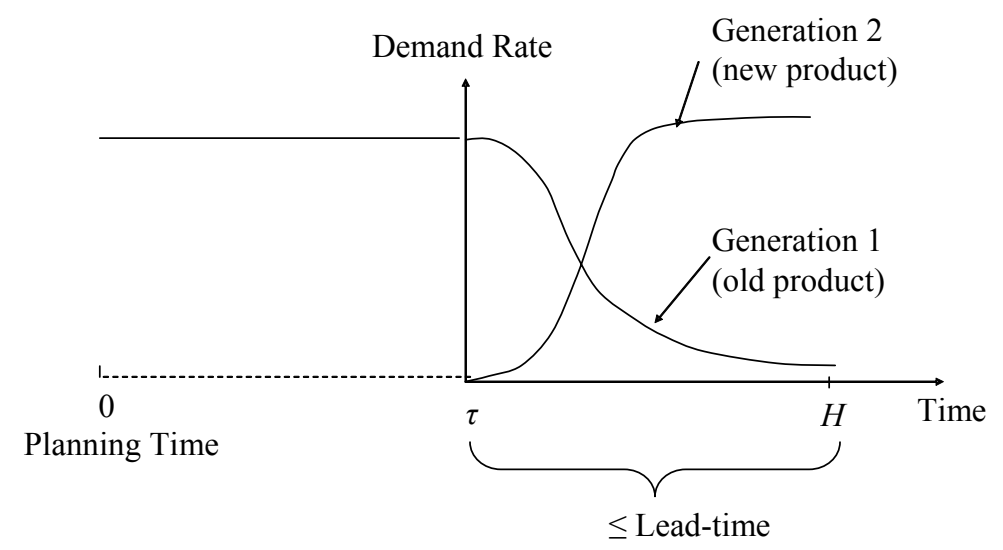

Figure 1. Demand Pattern during Product Transition

During the transition, if the old product runs out, the company can use the new product as a substitute. We assume that when the company decides to substitute, it provides the customer with a price discount off the price of the new product that is sufficient for the customer to accept the substitute. In addition to this discount, the company might incur a loss of goodwill for giving a customer his/her second choice. We do not differentiate between the discount and the loss of goodwill, but define the sum of the two as the substitution cost.

Although the new product is typically an improvement over the old product, many customers still prefer the old product in the transition stage. For instance, when the customer has an in- 
stalled base of the old product, the old product might be strongly preferred due to the increased service, maintenance and training costs associated with introducing a new version of the product. The customer might also prefer an old product to avoid exposure to technological risks associated with a new product. In these cases, the discount (plus the loss of goodwill) could very well be larger than any price difference of the two products.

We assume that customers are not aware of the company's inventory status before placing an order and therefore do not engage in any strategic behavior that takes into account a possible substitution. Specifically, a customer who intends to purchase a new product does not pretend to be a customer of the old product in order to receive the substitution discount.

Unmet demand is lost. Realistically, there may be cases when the company decides not to offer a substitution when the old product runs out, but the customer may, after learning that the old product runs out, come back to buy the new product instead. In this case unmet demand is not entirely lost. For simplicity, we ignore such behavior in this paper. However, we discuss in the conclusion a potential extension of the model that incorporates this effect.

We assume that any excess inventory of the old product is salvaged only after the transition. In the high-tech industry, the manufacturer can recoup about $10 \%$ of the original sales value of the product by selling its excess old product to a broker, who then sells the product in the secondary market. To avoid competing with its own product on the secondary market, a company would rarely salvage its excess inventory before the end of a transition. After the transition, the company generally views the secondary market as much less of a threat as the new product matures and the company stops shipping the old product. Hence we assume that salvaging the old product after the transition does not affect the sales of the new product.

We assume that the company continues to sell the new product after the transition ends and 
will be making replenishment orders during the transition period that by assumption do not arrive until after the transition period. Any leftover inventory of the new product at the end of the transition is not salvaged but is used to satisfy demand beyond the horizon. The company will adjust its replenishment orders placed during the transition period, based on the projected size of the leftover inventory. For our model, we approximate the salvage value of the new product inventory at the end of the transition period by the savings from reducing the first replenishment quantity received after time $H$; thus, the salvage value is the unit procurement cost of the new product at time $H$. This is an approximation since the amount leftover might be more than we would purchase in a single replenishment and we would need a more complex representation for the value of these leftover units. In this case, we could rank order the items and apply further incremental discount to each unit (Rosenfield 1989). The total salvage value would then be a nonlinear but concave function of the amount of leftover inventory at time $H$, and we expect all the proofs in this paper to remain valid.

\section{Deterministic Transition Start Date}

We start with the case when the new product release date (equivalently, the transition start date) is deterministic. Sometimes a product transition is driven by outside forces; for instance, a supplier sets an end-of-life for a component or the government issues new regulations for which the old product does not comply. In these cases, the company does not have much latitude in deciding when to start the transition, and thus the TSD is known and fixed.

Our objective is to find the starting inventory levels for both products, at time 0 . We use dynamic programming (DP) to formulate the problem as a discrete time model. Given that the demand is Poisson, we define the time unit such that the probability of more than one demand arrival (old or new) during one time unit is negligible. The time unit might correspond to a week, a 
day or an hour. Given the time unit, we define $\lambda_{1}(t)$ and $\lambda_{2}(t)$ to be the demand rates for the old and new product respectively. Throughout the paper, we use the subscript 1 to refer to the old product and the subscript 2 to refer to the new product. In each time unit $t$, the demand probability is $\lambda_{1}(t)$ for an old product and is $\lambda_{2}(t)$ for a new product; the probability of no demand is $1-$ $\lambda(t)$ where $\lambda(t)=\lambda_{1}(t)+\lambda_{2}(t)<1$. Without loss of generality, we assume that a demand (if any) always occurs at the beginning of a time unit.

\subsection{Substitution Decision}

We first note that if there were no demand substitution between the two products, inventory planning could be simplified to two independent problems: We can regard the transition period $[\tau, H]$ as a single selling-season with one order opportunity and find the quantities that satisfy the newsboy ratio for the old and new products respectively.

Since the old and new products share a common customer pool, risk pooling through substitution is possible. We assume that substitution is only relevant when the old product inventory runs out. We then need to decide for each demand for the old product whether or not to satisfy it with a unit of new product. This is a dynamic decision because it depends on the current inventory level of the new product and how far we are into the transition period. If we substitute, we avoid the lost sale of an old product and save some holding cost of a new product, but we may lose a sale of the new product if the new product inventory runs out before the transition ends. Therefore, we need to weigh the immediate benefit of substitution against the expected future loss from possible missed sales of the new product.

With a substitution, the company realizes the price of the new product $r_{2}$, net the substitution cost $g$, which includes a discount off the price of the new product plus any goodwill loss. We assume $g \geq 0$ to restrict the revenue from substitution to be no greater than a regular sale of the 
new product. In the extreme case, $g$ might be zero, indicating that the customers are willing to substitute the new product for the old product without discount or any loss of goodwill. In this case, a company will always substitute since it doesn't result in any loss. Alternatively a customer might be happy to take the new product at the price of the old when the new product is a higher-priced product, in which case $g=r_{2}-r_{1}$. However, if $g$ is large, a company might prefer to not fill old product demand. For example, Intel would rather save the new product for a customer who is willing to pay a $200 \%$ premium for a $10 \%$ performance boost from the new product, than sell it to the customer who wants the old product and is only willing to buy the new product at a significant discount.

Let $r_{i}$ be the selling price of product $i$ and $p_{i}$ be the penalty for a shortage of product $i$ in addition to the lost revenue. Hence $v_{i}=r_{i}+p_{i}$ is the total cost of a lost sale for product $i$. We let $x_{i}$ be the inventory level of product $i$ and $h_{i}$ the holding cost for product $i$ per unit time. $\delta$ is the discount factor per time unit. We denote by $V\left(x_{1}, x_{2}, t\right)$ the expected profit-to-go at time $t$ given that the inventory levels are $x_{1}$ and $x_{2}$. At any time $t$ during the transition, we can state the profit-to-go by the following recursion:

$$
\begin{array}{rlrl}
V\left(x_{1}, x_{2}, t\right) & =(1-\lambda(t))\left[-h_{1} x_{1}-h_{2} x_{2}+\delta V\left(x_{1}, x_{2}, t+1\right)\right] & \\
& +\lambda_{2}(t)\left[-h_{1} x_{1}-h_{2}\left(x_{2}-1\right)+r_{2}+\delta V\left(x_{1}, x_{2}-1, t+1\right)\right] & & \text { if } x_{1} \geq 1, x_{2} \geq 1 \\
& +\lambda_{1}(t)\left[-h_{1}\left(x_{1}-1\right)-h_{2} x_{2}+r_{1}+\delta V\left(x_{1}-1, x_{2}, t+1\right)\right] & \\
V\left(x_{1}, 0, t\right) & =(1-\lambda(t))\left[-h_{1} x_{1}+\delta V\left(x_{1}, 0, t+1\right)\right] & \\
+ & \lambda_{2}(t)\left[-h_{1} x_{1}-p_{2}+\delta V\left(x_{1}, 0, t+1\right)\right] & \\
+ & \lambda_{1}(t)\left[-h_{1}\left(x_{1}-1\right)+r_{1}+\delta V\left(x_{1}-1,0, t+1\right)\right] & \\
V\left(0, x_{2}, t\right) & =(1-\lambda(t))\left[-h_{2} x_{2}+\delta V\left(0, x_{2}, t+1\right)\right] & \\
+ & \lambda_{2}(t)\left[-h_{2}\left(x_{2}-1\right)+r_{2}+\delta V\left(0, x_{2}-1, t+1\right)\right] & \\
+ & \lambda_{1}(t) \max \left[-h_{2} x_{2}-p_{1}+\delta V\left(0, x_{2}, t+1\right),\right. & & \text { if } x_{2} \geq 1 \\
& \left.-h_{2}\left(x_{2}-1\right)+r_{2}-g+\delta V\left(0, x_{2}-1, t+1\right)\right] &
\end{array}
$$




$$
\begin{aligned}
V(0,0, t) & =(1-\lambda(t)) \delta V(0,0, t+1) \\
& +\lambda_{2}(t)\left[-p_{2}+\delta V(0,0, t+1)\right] \\
& +\lambda_{1}(t)\left[-p_{1}+\delta V(0,0, t+1)\right]
\end{aligned} \quad t=\tau, \tau+1, \tau+2, \ldots, H
$$

We define the terminal value $V\left(x_{1}, x_{2}, H+1\right)=s_{1} x_{1}+s_{2} x_{2}$ where $s_{i}$ is the salvage value of product $i$ at the end of the planning horizon. We use the DP formulation to prove our theoretical results, as well as to compute the optimal solutions.

The unstated assumption in equation (1) is that a company will never turn away customers when the product demanded is in stock: If a customer asks for product $i$ and there is inventory for product $i$, we will sell it. We make the following assumptions so as to facilitate the proofs.

$$
\begin{aligned}
& v_{1}+h_{1} \geq \delta s_{1} \\
& v_{2}+h_{2} \geq \delta s_{2} \\
& r_{2}-g+p_{1}+h_{2} \geq \delta s_{2} \\
& r_{2}-g+h_{2}-\delta s_{2} \leq r_{1}+h_{1}-\delta s_{1} \\
& r_{2}-g+\frac{h_{2}}{1-\delta} \leq r_{1}+\frac{h_{1}}{1-\delta}
\end{aligned}
$$

The assumptions (2)-(4) imply that it is better to sell a product than to salvage it. The assumptions (5) and (6) indicate that it is not worthwhile to substitute when there is inventory for the old product. Whereas there might be realistic cases when a company prefers to scrap the old product and offer only the new product, this is out of the scope of this paper.

Since the substitution decision is only relevant when the inventory of the old runs out, we can easily derive from equation (1) that the optimal policy is to substitute at time $t$ if and only if

$$
r_{2}+p_{1}-g+h_{2}>\delta\left[V\left(0, x_{2}, t+1\right)-V\left(0, x_{2}-1, t+1\right)\right] .
$$

Condition (7) has a simple interpretation: We substitute when the value from substitution (LHS) is larger than the discounted future loss from having one less unit of the new product (RHS).

Lemma $1 \alpha\left(x_{2}, t\right) \equiv V\left(0, x_{2}, t+1\right)-V\left(0, x_{2}-1, t+1\right)$ is non-increasing in $x_{2}$. 
Lemma 1 establishes that the value function $V\left(0, x_{2}, t\right)$ is concave in $x_{2}$ for all $t$. That is, there is decreasing marginal return from having more inventory of the new product. Lemma 1 and condition (7) lead to the optimality of a threshold policy.

\section{Proposition 1 (Threshold Substitution Policy)}

The optimal substitution policy is a time-varying threshold policy. There exists a threshold level $\bar{x}_{2}(t)$ such that when $x_{1}(t)=0$, it is optimal to substitute a new product to meet the demand for

the old product if the inventory level of the new product is at or above $\bar{x}_{2}(t)$; no substitution should be allowed when the new product inventory is below that level.

We define $\bar{x}_{2}(t)$ as the largest inventory level $x_{2}$ such that $\delta \alpha\left(x_{2}, t\right) \geq r_{2}+p_{1}-g+h_{2}$ holds. In the case when $\delta \alpha(1, t)<r_{2}+p_{1}-g+h_{2}$, we define $\bar{x}_{2}(t)=0$.

As $\alpha\left(x_{2}, t\right)$ is a function of $t, \bar{x}_{2}(t)$ will vary with $t$. Intuitively, we might expect that $\bar{x}_{2}(t)$ decreases in $t$ since the chance of running out and missing a sale of the new product should decline as time goes on. Indeed, this can be true, but only for certain special cases.

Proposition 2 With homogeneous Poisson demand, i.e., $\lambda_{1}(t)=\lambda_{1}$ and $\lambda_{2}(t)=\lambda_{2} \forall t \in(\tau, H)$, the substitution threshold $\bar{x}_{2}(t)$ is non-increasing in $t$.

Homogeneous Poisson demand implies that the expected demand for the old product drops instantly to some lower level after the new product is introduced and stays at that level throughout the transition period $[\tau, H]$, and that the expected demand for the new product jumps from zero to a constant level immediately after its release. Figure 2 shows how the threshold changes over time under homogeneous Poisson demand. Since the demand pattern during $[\tau$, $H]$ does not depend on the value of $\tau$, we plot the substitution threshold against the time elapsed since the TSD, i.e., $t-\tau$. Hence we only need the parameter value of $H-\tau$. When the new product inventory is above the curve, the optimal decision is to substitute using the new for the old; when 
the new product inventory is below the curve, the optimal decision is not to substitute and leave the order for the old product unfilled.

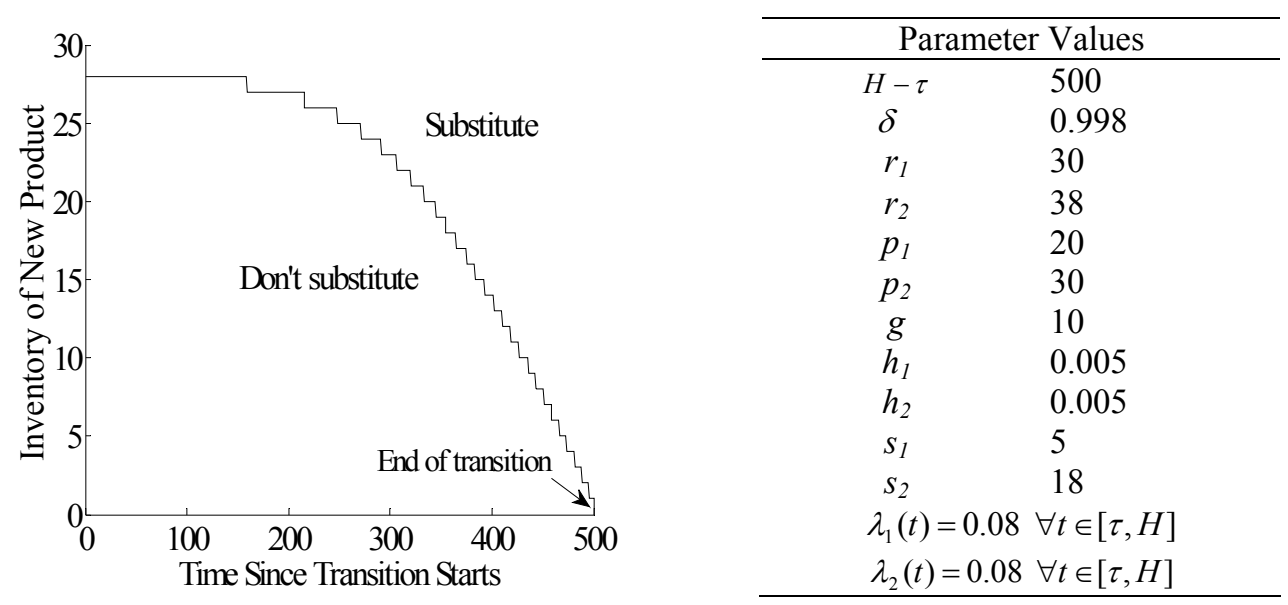

Figure 2. Substitution Threshold under Homogeneous Poisson Demand

The further into the transition period, the less time there is to sell a product; hence the motivation for substitution becomes stronger and the threshold decreases. This problem formulation also describes the optimal substitution policy for two substitutable products with stationary demands in general (not as a pair of overlapping generations of products).

Proposition 3 If the demand is non-homogeneous Poisson, and the holding cost and revenue discounting are negligible, i.e., $h_{1}=h_{2}=0$ and $\delta=1$, then $\bar{x}_{2}(t)$ is non-increasing in $t$.

The substitution decision is a tradeoff of the immediate revenue against possible future sales loss of the new product. The expected future loss depends on the time at which the new product sale might occur, because this time determines the revenue discounting applied to the sale as well as the holding cost incurred up to the sale. When we ignore holding cost and revenue discounting, this dependency vanishes; thus, the cost of substitution is only the expected revenue of a future sale, which is non-increasing in $t$. As a result, $\bar{x}_{2}(t)$ is non-increasing in $t$.

When the holding cost or revenue discounting is significant, $\bar{x}_{2}(t)$ need not be monotonic. 
We plot the demand rates $\lambda_{1}(t)=0.16 /\left(1+e^{0.025(t-\tau-250)}\right)$ and $\lambda_{2}(t)=0.16 /\left(1+e^{-0.025(t-\tau-250)}\right)$ in Figure 3(a). For these demand rates, we see in Figure 3(b) that the threshold increases during the early stage of the transition and then decreases later in the transition. That is, substitution is more favored at the beginning and the end of the transition. Again the horizontal axis in Figure 3 is $t-\tau$. All other parameter values are the same as in Figure 2.

(a) Demand

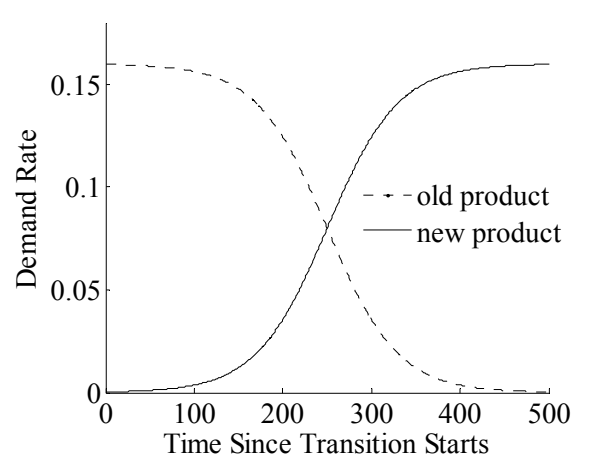

(b) Threshold

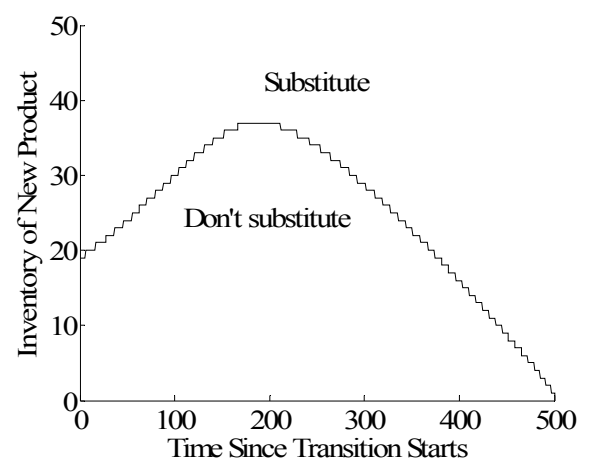

Figure 3. Substitution Threshold under Non-homogeneous Poisson Demand

Early in the transition, sales of the new product have not taken off, the optimal policy has a low threshold for substitution as it is more profitable to use new product inventory to meet demand for the old product, rather than hold this inventory until it can be used to meet new product demand later in the transition period. The immediate revenue from the substitution sale exceeds the expected discounted revenue from a new product sale, net of the holding cost. As the demand of the new product ramps up, the expected time to a sale of the new product decreases. This reduces the impact from the holding cost and revenue discount, and therefore, the substitution threshold increases. Later during the transition, the other dynamic starts dominating: Less and less time is left to sell, so we prefer to substitute a new product to meet old-product demand rather than risk not selling the inventory at all.

We establish this formally in Proposition 4 for the case of a constant aggregate demand rate. Proposition 4 If $\lambda_{1}(t)$ is non-increasing in $t$ and $\lambda_{2}(t)$ is non-decreasing in $t$, and 
$\lambda_{1}(t)+\lambda_{2}(t)=\lambda, \forall t$, then $\bar{x}_{2}(t)$ is a unimodal function of $t$ : it is non-decreasing in $t$ before a certain time $\hat{t}$ and non-increasing in t after $\hat{t}$.

\subsection{Optimal Initial Inventories}

So far we have not accounted for the procurement and production costs since these can be considered sunk costs in making the substitution decision. However, we need to consider these costs when we solve for the optimal initial quantities. Under the assumption of linear cost, we denote the unit cost of product $i$ by $c_{i}$ and define the net profit as

$$
N V\left(x_{1}, x_{2}\right) \equiv V\left(x_{1}, x_{2}, 0\right)-c_{1} x_{1}-c_{2} x_{2} .
$$

Using equation (1) we show that $V\left(x_{1}, x_{2}, t\right)$ is jointly concave in $x_{1}$ and $x_{2}$.

Proposition $5 V\left(x_{1}, x_{2}, t\right)$ is jointly concave in $x_{1}$ and $x_{2}$.

It follows that $N V$ will also be jointly concave in $x_{1}$ and $x_{2}$. Hence we can easily find the optimal starting inventories by a simple search.

The implication of the joint concavity of $V$ with respect to the inventory levels of both products is twofold. First, there is decreasing marginal return to more inventory. Second, there is decreasing marginal value from substitution: The value of an additional unit of new product is higher (lower) when the old product inventory is low (high); the value of an additional unit of old product is higher (lower) when the new product inventory is low (high).

\section{Stochastic Transition Start Date}

A new-product launch involves many sequential steps including engineering development and testing, material sourcing, manufacturing, and delivery. With inherent uncertainties in each step, the actual release date often fluctuates dramatically. If the new product development slips and the inventory of the old product is depleted, supply gaps can result. Consequently, a much larger supply cushion of the old product is needed compared to the case of a deterministic TSD. 


\subsection{Optimal Planning Quantities under Stochastic TSD}

Let $\tau$ be the TSD. We assume now that the TSD is stochastic, with an upper and a lower bound $\bar{\tau}$ and $\underline{\tau}$ respectively, and with $q(\tau=k)$ representing the probability that $\tau=k$. . As before, we make planning decisions and place orders at time zero for the planning horizon $[0, H]$. In addition, we assume that the firm completes the transition by the fixed time $H$, independent of the value of $\tau$, that is, at time $H$, the demand for the old product is negligible, the company stops selling the old product and salvages any leftover inventory.

Let $W\left(\tau, x_{1}, x_{2}, t\right)$ denote the profit-to-go from time $t$ to $H$ for a given realization of $\tau$, where $x_{1}$ and $x_{2}$ are the inventory levels of the old and new product at time $t$. Since the transition has not started before $\tau, \lambda_{I}(t)=\lambda_{1}$ and $\lambda_{2}(t)=0$ for $t \in[0, \tau]$. Thus, for any $t \in[0, \tau]$, we have:

$$
\begin{aligned}
W\left(\tau, x_{1}, x_{2}, t\right) & =\left(1-\lambda_{1}\right)\left[-h_{1} x_{1}+\delta W\left(\tau, x_{1}, x_{2}, t+1\right)\right] \\
& +\lambda_{1}\left[-h_{1}\left(x_{1}-1\right)+r_{1}+\delta W\left(\tau, x_{1}-1, x_{2}, t+1\right)\right]-h_{2} x_{2} \quad \text { if } x_{1} \geq 1 \\
W\left(\tau, 0, x_{2}, t\right)= & \left(1-\lambda_{1}\right) \delta W\left(\tau, 0, x_{2}, t+1\right) \\
& +\lambda_{1}\left[-p_{1}+\delta W\left(\tau, 0, x_{2}, t+1\right)\right]-h_{2} x_{2}
\end{aligned}
$$

We define the terminal value $W\left(\tau, x_{1}, x_{2}, \tau\right) \equiv V\left(x_{1}, x_{2}, \tau\right)$, as given in Section 4. Thus $W\left(\tau, x_{1}, x_{2}, 0\right)$ is the expected profit from time 0 to $H$ for a given TSD $\tau$. To obtain the expected profit for a stochastic TSD, we weight each $W\left(\tau, x_{1}, x_{2}, 0\right)$ by the probability $q(\tau)$ :

$$
E W\left(x_{1}, x_{2}\right)=\sum_{\tau=\underline{\tau}}^{\bar{\tau}} q(\tau) W\left(\tau, x_{1}, x_{2}, 0\right) .
$$

Finally, we subtract the procurement costs from $W$ to obtain the net profit

$$
N W\left(x_{1}, x_{2}\right)=E W\left(x_{1}, x_{2}\right)-c_{1} x_{1}-c_{2} x_{2}
$$

Since $V\left(x_{1}, x_{2}, t\right)$ is jointly concave in $x_{1}$ and $x_{2}$, it follows that $N W\left(x_{1}, x_{2}\right)$ is also jointly concave in $x_{1}$ and $x_{2}$. Therefore, we can easily find the optimal initial inventories $\left(x_{1}, x_{2}\right)$ to cover 
demand during $[0, H]$, given the procurement $\operatorname{cost} c_{1}$ and $c_{2}$.

Proposition $6 N W\left(x_{1}, x_{2}\right)$ is jointly concave in $x_{1}$ and $x_{2}$.

Often at time zero we will have an existing inventory of the old product $x_{1}^{0}$. For this case we define $N W\left(x_{1}, x_{2}, x_{1}^{0}\right)$ to be the net profit only for $x_{1} \geq x_{1}^{0}$ because we cannot salvage the old product until the end of the transition period. Thus we rewrite the objective function in (10) as:

$$
N W\left(x_{1}, x_{2}, x_{1}^{0}\right)=E W\left(x_{1}, x_{2}\right)-c_{1}\left(x_{1}-x_{1}^{0}\right)-c_{2} x_{2} \quad \forall x_{1} \geq x_{1}^{0}
$$

\section{Proposition 7 (Order-up-to Policy)}

If the inventory position at the time of planning is $x_{1}^{0}$, then the optimal order quantities are $\left(x_{1}^{*}-x_{1}^{0}, x_{2}^{*}\right)$ if $x_{1}^{0}<x_{1}^{*}$ and $\left(0, x_{2}\left(x_{1}^{0}\right)\right)$ if $x_{1}^{0} \geq x_{1}^{*}$, where $\left(x_{1}^{*}, x_{2}^{*}\right)$ maximizes $N W\left(x_{1}, x_{2}\right)$ as de-

fined in equation (10). $x_{2}\left(x_{1}^{0}\right)$ is the optimal quantity of the new product given that the planning quantity for the old product is set to $x_{1}^{0}$. In addition, if $x_{1}^{0} \geq x_{1}^{*}$, then $x_{2}\left(x_{1}^{0}\right) \leq x_{2}^{*}$

The implications of Proposition 7 are simple: if the company has more inventory of the old product than needed, the optimal policy is to order no old product and plan the same or less for the new product than it would have when the existing inventory is not considered. When we have too much old product, the value of substitution decreases and we require less new product.

\subsection{Comparison to the Optimal Planning Decisions under Deterministic TSD}

We use numerical examples to explore the effect of variability in the TSD, as well as the sensitivity of the optimal solution to the substitution cost and the salvage value of the new product.

For each of the above parameters, we allow three values, signifying a small, medium, or large value. Specifically, we set the salvage value of the new product to the unit cost of the initial order, $c_{2}$ (large), or $80 \%$ of $c_{2}$ (medium), or $50 \%$ of $c_{2}$ (small). Similarly, we set the substitution cost: $r_{2}-0.8 r_{1}$ (large), $r_{2}-r_{1}$ (medium), and $r_{2}-1.1 r_{1}$ (small). That is, we achieve an actual rev- 
enue of $80 \%$ of $r_{1}, 100 \%$ of $r_{1}$, and $110 \%$ of $r_{1}$, respectively. We model the TSD as a uniformlydistributed discrete random variable with mean of 500. . For the high variance case, $\tau$ is uniformly distributed on the interval $[250,750]$ with a grid of 25 ; in the medium variance case, the interval is $[400,600]$ with a grid of 25 ; in the low variance case, $\tau$ is deterministic, equal to 500 . We let $\delta=1$ to exclude the effect from revenue discounting. Table 1 shows the optimal solution for each of the 27 scenarios. Other parameter values are shown in Table 2.

\begin{tabular}{c|c|c|c|c|c|c|c}
\hline & $\begin{array}{c}\text { Standard de- } \\
\text { viation of TSD }\end{array}$ & $\begin{array}{c}\text { Substitution } \\
\text { cost }(\mathrm{g})\end{array}$ & $\begin{array}{c}\text { Salvage val- } \\
\text { ue }\left(s_{2}\right)\end{array}$ & $\begin{array}{c}\text { Optimal } \\
x_{1}\end{array}$ & $\begin{array}{c}\text { Optimal } \\
x_{2}\end{array}$ & $x_{1}+x_{2}$ & $\begin{array}{c}\text { Optimal } \\
\text { Expected Profit }\end{array}$ \\
\hline 1 & 0 & 5 & 9 & 94 & 82 & 176 & 2180.6 \\
2 & 0 & 5 & 14.4 & 93 & 86 & 179 & 2236.4 \\
3 & 0 & 5 & 18 & 93 & 89 & 182 & 2283.2 \\
4 & 0 & 8 & 9 & 101 & 75 & 176 & 2160.6 \\
5 & 0 & 8 & 14.4 & 99 & 80 & 179 & 2210.0 \\
6 & 0 & 8 & 18 & 97 & 86 & 183 & 2254.2 \\
7 & 0 & 14 & 9 & 104 & 73 & 177 & 2138.1 \\
8 & 0 & 14 & 14.4 & 104 & 76 & 180 & 2181.9 \\
9 & 0 & 14 & 18 & 103 & 80 & 183 & 2220.4 \\
10 & 25.8 & 5 & 9 & 95 & 81 & 176 & 2144.8 \\
11 & 25.8 & 5 & 14.4 & 95 & 84 & 179 & 2197.7 \\
12 & 25.8 & 5 & 18 & 96 & 87 & 183 & 2241.7 \\
13 & 25.8 & 8 & 9 & 97 & 80 & 177 & 2116.6 \\
14 & 25.8 & 8 & 14.4 & 97 & 83 & 180 & 2169.5 \\
15 & 25.8 & 8 & 18 & 97 & 86 & 183 & 2213.6 \\
16 & 25.8 & 14 & 9 & 100 & 79 & 179 & 2067.7 \\
17 & 25.8 & 14 & 14.4 & 100 & 82 & 182 & 2121.0 \\
18 & 25.8 & 14 & 18 & 100 & 85 & 185 & 2165.4 \\
19 & 60.6 & 5 & 9 & 98 & 87 & 185 & 1808.7 \\
20 & 60.6 & 5 & 14.4 & 98 & 93 & 191 & 1904.1 \\
21 & 60.6 & 5 & 18 & 100 & 98 & 198 & 1984.9 \\
22 & 60.6 & 8 & 9 & 99 & 87 & 186 & 1778.1 \\
23 & 60.6 & 8 & 14.4 & 100 & 93 & 193 & 1874.3 \\
24 & 60.6 & 8 & 18 & 101 & 98 & 199 & 1956.3 \\
25 & 60.6 & 14 & 9 & 101 & 87 & 188 & 1719.8 \\
26 & 60.6 & 14 & 14.4 & 103 & 92 & 195 & 1818.3 \\
27 & 60.6 & 14 & 18 & 104 & 98 & 202 & 1902.8 \\
\hline
\end{tabular}

Table 1. Sensitivity Analysis 


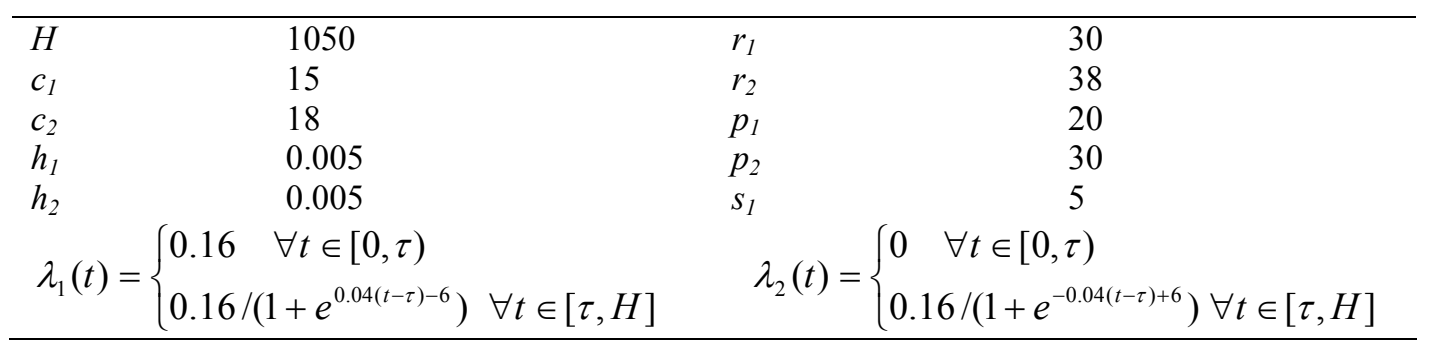

Table 2. Parameter Values

We observe from Table 1 that the total expected profit deteriorates as the TSD becomes more variable, ceteris paribus. To illustrate this, we show in Figure 4 how the total profit changes with the variability of the TSD for $g=10, s_{2}=18$, and other parameter values as shown in Table 2 . The demand rates behave similar to Figure 3(a). We define the total expected profit with "no substitution" as the profit originating from the heuristic policy where the firm never substitutes demand for the old product, i.e., the firm always picks the first term in the "max" expression of equation (1). We observe that the value from substitution (the gap between the two curves in Figure 4) increases as the TSD becomes more variable.

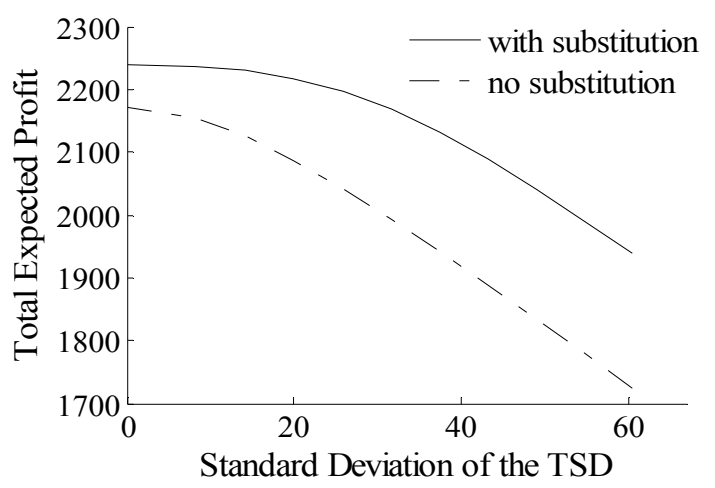

Figure 4. The Total Expected Profit vs. Uncertainty in TSD

The total quantity for the two products increases with the TSD uncertainty, as expected. However, we do not observe a monotonic trend of the optimal quantity for each product with respect to the TSD uncertainty. In fact, the optimal quantity for either product may initially fall and then increase as the TSD variability changes from low to medium, and then to high (compare cases 8,17 and $26, g=14$ and $s_{2}=14.4$, for the optimal quantity of the old product and cases 3 , 
12 and $21, g=5$ and $s_{2}=18$, for the optimal quantity of the new product). In Figure 5, we illustrate this non-monotonic pattern by graphing the optimal quantities for a range of TSD variability for a fixed salvage value of the new product $\left(s_{2}=18\right)$. It may seem counterintuitive that, in the case when $g=5$, one may need to reduce the quantity of the new product as variability grows, precisely when substitution would be most beneficial (a small substitution cost).

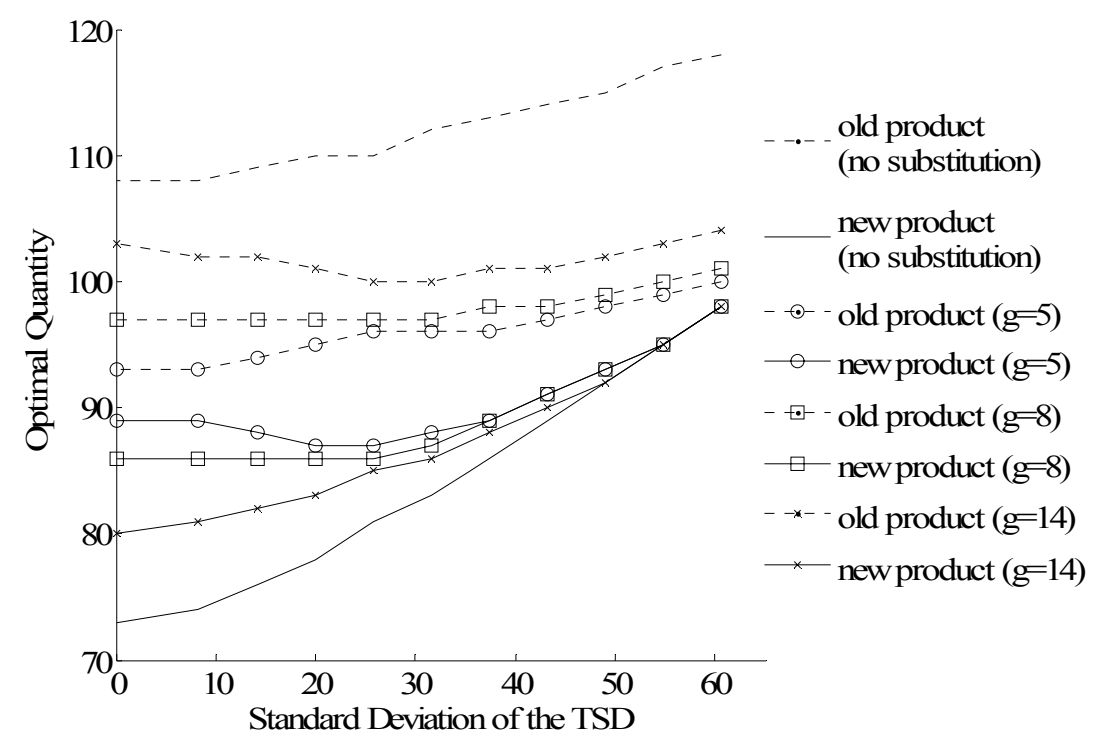

Figure 5. Optimal Quantities vs. Standard Deviation of the TSD

To explain this observation, we note first that without substitution, the necessary supply cushion for each product increases in the TSD uncertainty. We refer to this extra supply of inventory as the "basic cushion". While the new product consists of a "basic cushion" to protect against TSD uncertainty, it also includes a "substitution cushion" (shown as the gap between the plain solid curve and each marked solid curve in Figure 5) that allows substitution of the old product. However, there is risk pooling between the "basic cushion" and the "substitution cushion" of the new product, which reduces the "substitution cushion" as the variability of the TSD increases. The two factors, "basic cushion" and "substitution cushion" change in opposite directions with respect to TSD uncertainty, contributing to the non-monotonic pattern. 
Another consideration is the affect that TSD variability has on the amount of old product inventory needed before the transition starts. An increase in TSD variability increases the variability of the old-product demand prior to the TSD and hence the need for old product inventory. This additional inventory reduces the need for substitution during the transition period. While we might expect more TSD variability to always result in more substitutions, this need not be the case. As shown in Figure 6, when the substitution cost is small, more TSD variability results in fewer substitutions, due to the need to stock more old-product inventory for the demand variability in the interval $[0, \tau]$. Nevertheless, for any given variability level, more substitution is expected with a smaller $g$.

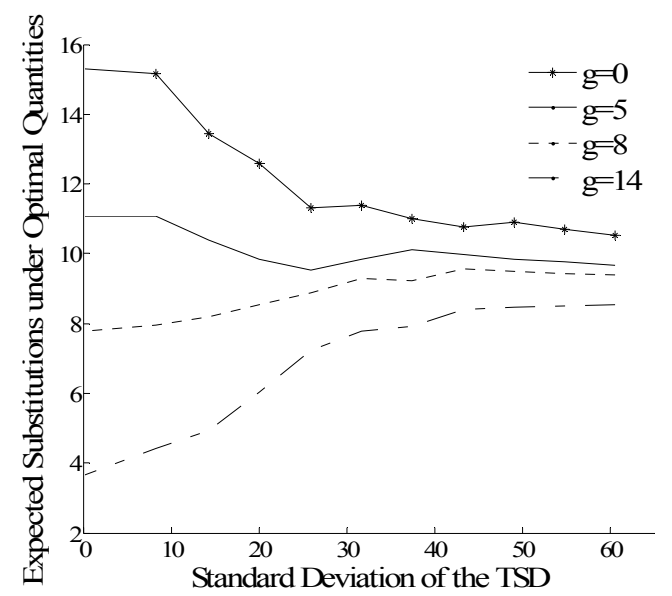

Figure 6. Expected Substitutions vs. Standard Deviation of the TSD

From Table 1, as well as Figure 5, we observe that the optimal quantity for the old (new) product is non-decreasing (non-increasing) in the substitution cost, as expected. The higher the substitution cost, we have less substitution; thus we need more inventory of the old product and less inventory of the new product.

The optimal quantity for the new product increases significantly in its salvage value, or equivalently, its future replacement cost. One implication is the importance of predicting how the new product cost decreases over time due to production learning and economy of scale. 
The optimal quantity for the old product may increase or decrease in the new product's salvage value. For example, in cases $4-6$, the optimal $x_{1}$ decreases as $s_{2}$ changes from low to medium and then to high, whereas in cases 22-24, the optimal $x_{1}$ increases as $s_{2}$ increases. Since we can substitute the new product for the old, we are effectively pooling the demand of the two products. Thus a larger salvage value for the new product reduces both the underage cost for the old product through substitution, and the risk of excess for the old product in that the company can dynamically reduce substitution. Therefore, depending on which of the two competing dynamics dominates, the optimal quantity of the old product may increase or decrease in the salvage value of the new product.

To see the profit impact of the optimal policy, we compare the optimal policy with other simple alternatives. Figure 7 shows the profit realized using the Newsboy quantities and naïve substitution policies that either substitute whenever the old product runs out or never substitute at all. We use the same parameter values as in Figure 4. Clearly both alternative policies fare worse than the optimal policy, especially at higher variation levels of the TSD.

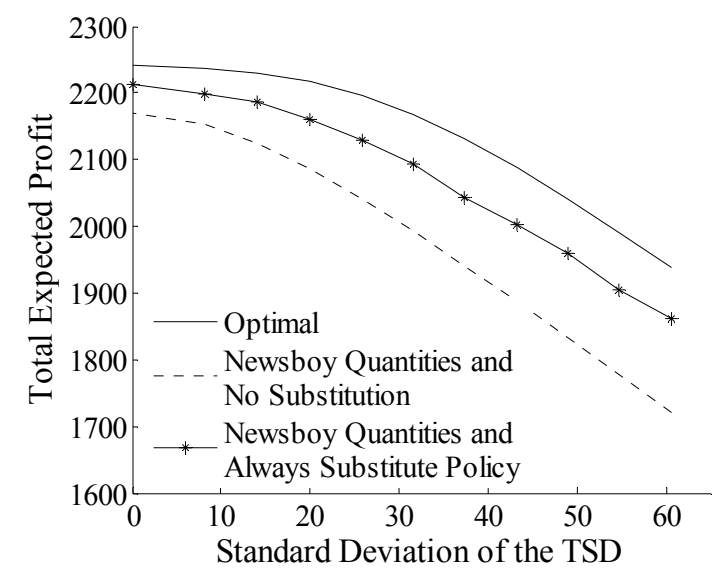

Figure 7. Comparison with Newsboy Quantities and Naïve Substitution Policies

\section{Determine the Optimal TSD}

Companies can sometimes delay the release of the new product to postpone the transition. The 
decision to delay involves a tradeoff between selling the excess inventory of the old product, and obtaining higher margins from selling the new product. Figure 8 illustrates the impact of a delay. Delaying the new product release from $\tau$ to $\tau^{\prime}$ increases the period of time that the old product is being sold and delays the sales of the new product. We use $L$ to denote the duration of the transition and $H$ the end of the planning horizon. We assume that both $H$ and $L$ are given fixed values

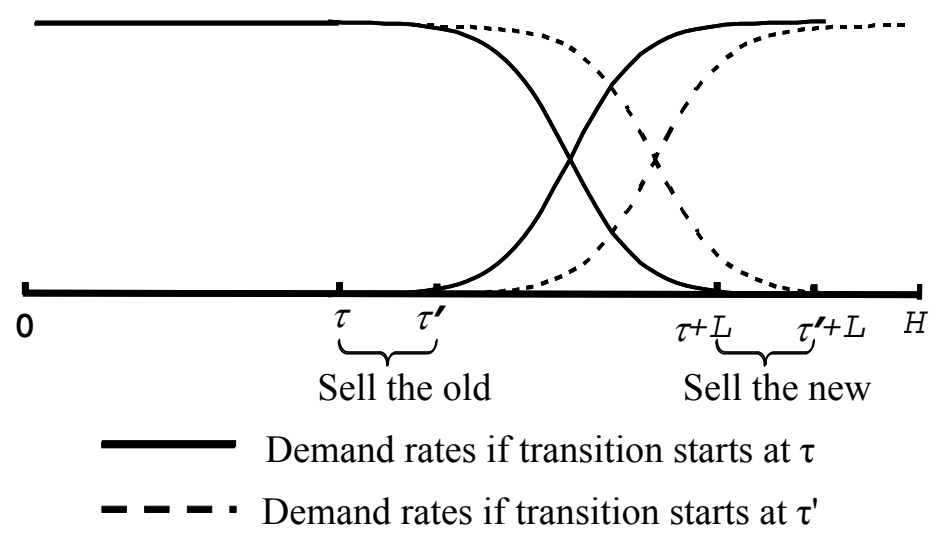

Figure 8. Impact of Delaying the TSD from $\tau$ to $\tau^{\prime}$

We assume that the decision of $\tau$ does not affect the subsequent demand pattern or the duration of the transition. This is a simplification of a situation that encompasses a number of factors. Often the timing of product release affects the market shares for the new product, especially in industries with intense competition (Wilson and Norton 1989, Souza et al. 2004, Savin and Terwiesch 2005). For example, in the race of video game consoles, Sony PlayStation2 obtained a $70 \%$ market share by entering the market a year earlier than Microsoft's Xbox and Nintendo's GameCube. In these extreme cases, competition is often the primary factor for determining the new product release date. Further, in practice, many other factors could affect the decision of the release date of a new product such as engineering and manufacturing constraints, time since last product upgrade, and so on. In this paper, we do not intend to address every facet of the problem, but instead choose to focus on one particular tradeoff between reducing excess inventory of 
the old product and capturing the margin premium of the new product. Our goal is to examine the impact of such an operational consideration, but with the recognition that this is not the only determinant for the new product introduction date. In this section, we also explore how the speed of the transition affects the decision of delaying a new product release.

If we view the substitution decision as a contingency strategy for a shortage of the old product, the delay of a new product release addresses the opposite situation - an excess inventory of the old product. Delaying the new product allows the company to sell more of the old product to alleviate the excess problem, albeit at the cost of reduced revenue from the new product.

We consider a finite horizon $[0, H]$. Given an initial inventory of the old product $x_{1}^{0}$, we wish to determine at time zero both the TSD, and the order-up-to levels for the old and new products. We assume that we can set TSD within a deterministic time window $[\underline{\tau}, \bar{\tau}]$ and there is no uncertainty once it is set. We assume that the transition finishes before time $H$ for any choice of the TSD; that is, by time $H$ the demand for the old product is negligible. The objective is to choose the optimal TSD to maximize the expected net profit over the planning horizon $[0, H]$, given an initial inventory. In practice, such a static policy makes sense as many coordinated activities beyond just the inventory process depend on knowing the start date as best as possible. However, one might conceive of a dynamic policy in which we continue to adjust the TSD based on the demand realizations for the old product over some time interval.

We show in equation (9) how to obtain the total expected profit for $[0, H]$ for a given TSD $\tau$ and give the optimal order policy in Proposition 7. Hence we can obtain the optimal choice of $\tau$, as well as the optimal quantities of $x_{1}$ and $x_{2}$ through a search algorithm.

Figure 9 illustrates the dependency of the optimal TSD on $x_{1}^{0}$ for the given parameter values. The demand and all other parameters are as given in Table 2. 
(a) Optimal TSD

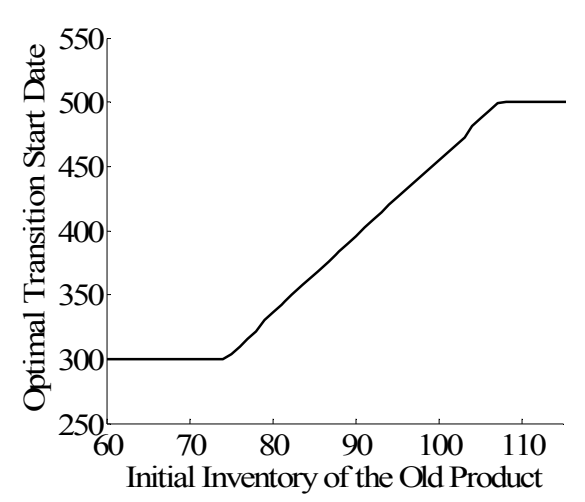

(b) Optimal Quantities

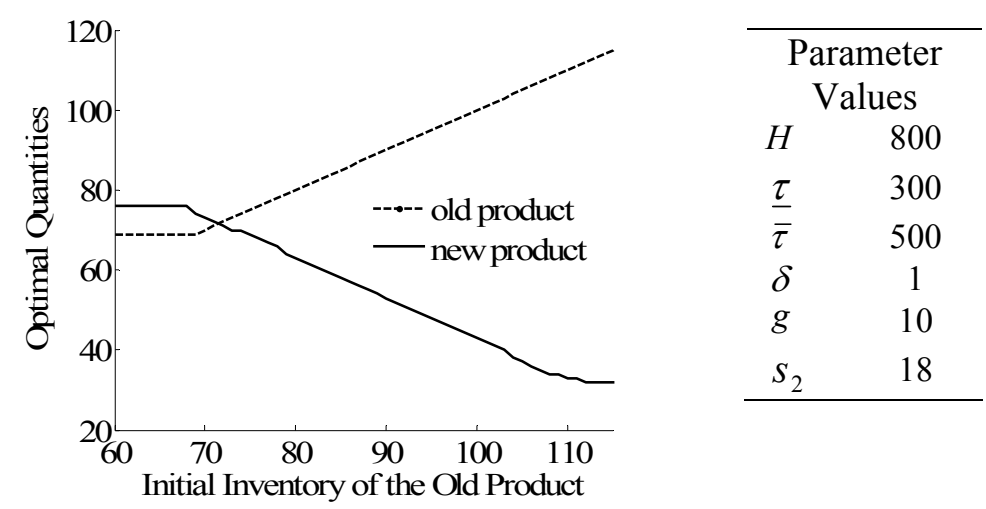

Figure 9. Optimal TSD and Quantities vs. Initial Inventory of the Old Product

The optimal TSD follows a threshold pattern: If the initial inventory $x_{1}^{0}$ is below a threshold $\bar{x}_{1}$, no delay is necessary, i.e., $\tau=\underline{\tau}$; otherwise, the optimal policy prescribes a delay of the new product release that increases in the initial inventory. If the initial inventory of the old product is between 0 and 68 , we order 75 units for the new product, order up to 68 for the old product, and release the new product on the scheduled release date 300 . For initial inventory between 68 and 74, no delay is necessary, we order no old product, and we order less new product. For inventory above 74, a delay is necessary and the optimal delay increases with the initial inventory level. Again no order is necessary for the old product and the number of units of new product to purchase decreases with the old product inventory. We note that the threshold quantity for delay (74) is greater than 68 , the ideal quantity for the old product when there is no delay and no prior inventory commitment. This is because to obtain the latter, we need to consider the variable product cost. However, to obtain the optimal delay, the costs of committed inventory are sunk.

In Figure 10, we show how the optimal TSD delay varies with the rate of the product replacement. We specify the parameter $k$ for the demand equations of the two products

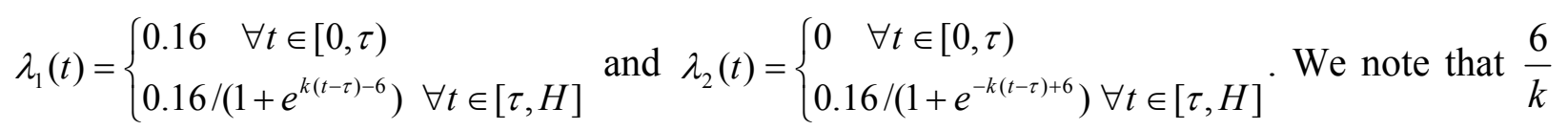


is the time elapsed since the transition when the two products reach equal demand share (Fisher and Pry 1971); thus the parameter $k$ represents the speed of the transition.

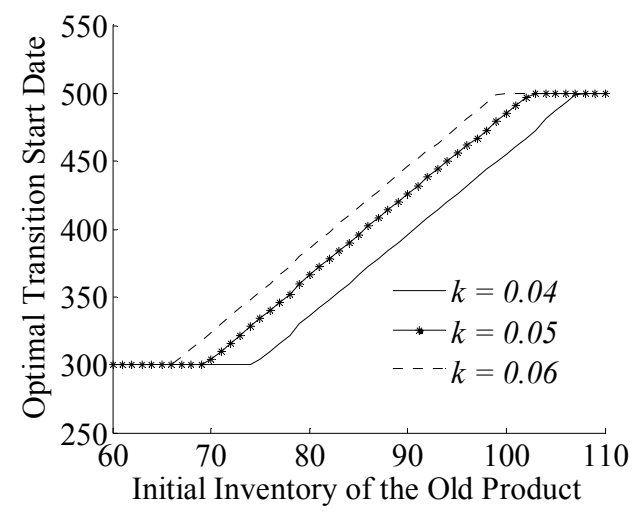

Figure 10. Optimal Delay vs. Rate of Transition

The faster the transition, the lower is the delay threshold. If we expect the transition to be fast, it is more likely that we will face the excess problem and therefore it would seem that the optimal strategy is to delay the new product release. However, this can be deceiving as a fast transition often implies that the new product is well-received by the market and that it will most likely draw a large margin. Thus a more reasonable strategy would be to consider changes in both parameters simultaneously. We leave it for future research to develop the analytical relationship between the rate of the transition and the price of the new product so as to explore the combined effect on the optimal delay strategy.

\section{Conclusions}

In this paper we solve an inventory planning problem for inter-generational product transitions when there is no replenishment opportunity during the transition period. We allow substitutions using the new product when the old product runs out. We show that the optimal substitution decision is a time-varying threshold policy with the substitution threshold $\bar{x}_{2}(t)$ increases in $t$ before a certain time $\hat{t}$ and decreases in $t$ after $\hat{t}$. We establish for both a deterministic and sto- 
chastic TSD that the total discounted net profit is a jointly concave function of the inventories for the old and new products. We can thus obtain the optimal planning quantities by a simple search.

We find that substitution reduces the need to hold the old product and can increase the profitability over the transition. Further, as the future replacement value of the new product increases, a company should increase the initial amount of the new product, which allows for more substitution and thus decreases the amount of the old product to hold. That said, substitution does not help a company when it runs out of the old product before a new product is ready. In the case of Blofeld, the company underestimated the time it took to develop and introduce the new product and did not have enough supply "cushion" for its old product. For them, the only remedy was to get the new product out as soon as possible to reduce the supply gap.

Our results indicate that TSD uncertainty can produce competing effects on the planning stocks. Whereas the total stock increases with increased TSD variability, the division between the old and new product inventory is not monotonic. Increasing variability results in more variable demand both prior to the transition and during the transition period. This results in pressure to hold both more new and old product inventory. Yet with more new product inventory there would seem to be more opportunity for substitution, which reduces the need for old product inventory in the transition period. Meanwhile, with more old-product inventory needed prior to the transition, the need for substitution during the transition period lessens, which puts downward pressure on the new product inventory. These countervailing forces lead to non-monotonic behavior as the TSD variability increases. In fact, our result suggests that, counter to our intuition, growing TSD uncertainty need not lead to more substitutions or more new product inventories.

Further, we have shown how to determine the optimal delay for a new product release when a company faces potential excess inventory of the old product. In essence, one should try to ad- 
dress excess inventory in the old product first through the initial stock decisions, and if that is not sufficient, subsequently delaying the release of the new product. In the Sultan transition example, the delay was warranted as the company foresaw a serious excess problem and to some extent the delay was a means for damage control.

A key assumption in our model is that there is no replenishment opportunity during the transition. This need not be true in contexts in which the transition period may take longer. While it is often difficult to replenish the old product after the last buy decision, there may be reordering opportunities for the new product during the transition. We would like to explore in future research the optimal inventory policy in these cases.

Also implicit in our model is that the product prices stay constant throughout the transition. This is not always the case in practice. In fact, pricing is an important tool for demand management. During product transitions, product managers often can manipulate the price gap of the two products in order to push a particular product. Li and Graves (2007) present a dynamic pricing model that examines the pricing decisions during a product transition. In future research, one may consider combining a pricing model with the planning problem.

We have assumed that a demand of the old product is lost if the company runs out of the old product and decides not to offer substitution. In reality, customers may come back and purchase the new product instead. One way to include this behavioral impact on demand is to allow only a portion of the unmet old product demand to be lost. We conjecture that most of the analytical results remain valid with this extension; however, we leave this extension for future research.

Acknowledgement: The authors are grateful to Professor Charles Fine for providing support and feedback on this research. We thank the referees, the associate editor and department editor for their helpful and constructive feedback on two earlier versions of this paper. We also thank Justin O'Connor and Alan Miano for sharing their industry knowledge. The second author acknowledges the support from the Singapore-MIT Alliance. 


\section{References}

Bassok, Y., R. Anupindi, R. Akella. 1999. Single-period multiproduct inventory models with substitution. Operations Research 47(4) 632-642.

Billington, C., H.L. Lee, C.S. Tang. 1998. Successful strategies for product rollovers. Sloan Management Review 39(3) 23-30.

Bitran, G.R., S. Dasu. 1992. Ordering policies in an environment of stochastic yields and substitutable demands. Operations Research 40(5) 999-1017.

Fisher, J.C., R.H. Pry. 1971. A simple substitution model of technological change. Technological Forecasting and Social Change 3 75-88.

Goyal, S.K., B.C. Giri. 2001. Recent trends in modeling of deteriorating inventory. European Journal of Operational Research 134(1) 1-16.

Hopman, J.W. 2005. Managing uncertainty in planning and forecasting. Intel Technology Journal, 9(3) 175-183.

Jain, K., E.A. Silver. 1994. Lot sizing for a product subject to obsolescence or perishability. European Journal of Operational Research 75(2) 287-295.

Kalpakam, S., K.P. Sapna. 1994. Continuous review (s,S) inventory system with random lifetimes and positive leadtimes. Operations Research Letters 16(2) 115-119.

Krishnamoorthy, A., T.V. Varghese. 1995. Inventory with disaster. Optimization 35(1) 85-93.

Li, H., S.C. Graves. 2007. Pricing decisions during inter-generational product transition. Working paper.

Mahajan, S., G. van Ryzin. 2001. Stocking retail assortment under dynamic consumer substitution. Operations Research 49(3) 334-351.

Parlar, M., S.K. Goyal. 1984. Optimal ordering decisions for two substitutable products with 
stochastic demands. Opsearch 21(1) 1-15.

Pasternack, B.A. Z. Drezner. 1991. Optimal inventory policies for substitutable commodities with stochastic demand. Navel Research Logistics 38(2). 221-240.

Rao, U.S., J.M. Swaminathan, J. Zhang. 2004. Multi-product inventory planning with downward substitution, stochastic demand and setup costs. IIE Transactions 36(1) 59-71.

Ravichandran, N. 1995. Stochastic analysis of a continuous review perishable inventory system with positive lead time and Poisson demand. European Journal of Operational Research 84(2) 444-457.

Rosenfield, D.B. 1989. Disposal of excess inventory. Operations Research 37(3) 404-409.

Rosenfield, D.B. 1992. Optimality of myopic policies in disposing excess inventory. Operations Research 40(4) 800-803.

Saenz, A., B. Damodaran, C. Wu, E. Portzline, J. Shim, M. Keck, M. Habersack, S. Rardin. 2005. Cash-to-cash cycle time improvement initiatives at Lexmark. http://www.cscmp.org/Downloads/public/Resources/Lexmark.pdf

Savin, S., C. Terwiesch. 2005. Optimal product launch times in a duopoly: Balancing life-cycle revenues with product cost. Operations Research 53(1), 26-47.

Smith, S.A., N. Agrawal. 2000. Management of multi-item retail inventory systems with demand substitution. Operations Research 48(1) 50-64.

Song, J., P.H. Zipkin. 1996. Managing inventory with the prospect of obsolescence. Operations Research 44(1) 215-222.

Souza, G.C., B.L. Bayus, H.M. Wagner. 2004. New-product strategy and industry clockspeed. Management Science 50(4) 537-549.

Wilson, L.O, J.A. Norton. 1989. Optimal entry timing for a product line extension. Marketing 
Science 8(1) 1-17.

Wilhelm, W.E., K. Xu. 2002. Prescribing product upgrades, prices and production levels over time in a stochastic environment. European Journal of Operational Research 138(3) 601621. 


\section{Proofs}

\section{Proof of Lemma 1}

We prove this by induction.

First note that $\alpha\left(x_{2}, t\right)$ non-increasing in $x_{2}$ is equivalent to $V$ being discretely concave.

For ease of exposition, we also define $\alpha(0, t) \equiv\left(v_{2}+h_{2}\right) / \delta$. From the terminal value definition, $V\left(0, x_{2}, \tau+T+1\right)=s_{2} x_{2}$, thus $\alpha\left(x_{2}, \tau+T\right)=s_{2}$. From assumption (3), $\alpha(1, \tau+T)<\alpha(0, \tau+T)$. Therefore, $\alpha\left(x_{2}, \tau+T\right)$ is non-increasing in $x_{2}$.

Assume for induction that $\alpha\left(x_{2}, t\right)$ is non-increasing in $x_{2}$. Then condition (7) implies that $\exists \bar{x}_{2}(t)$ s.t. it is optimal to substitute at $t$ if and only if $x_{2}>\bar{x}_{2}(t)$. Then from equation (1), we have:

$$
\begin{gathered}
\alpha\left(x_{2}, t-1\right)=(1-\lambda(t))\left[-h_{2}+\delta \alpha\left(x_{2}, t\right)\right]+\lambda_{2}(t)\left[-h_{2}+\delta \alpha\left(x_{2}-1, t\right)\right] \\
+\lambda_{1}(t) \begin{cases}-h_{2}+\delta \alpha\left(x_{2}, t\right) & \text { if } x_{2} \leq \bar{x}_{2}(t) \\
-h_{2}+\delta \alpha\left(x_{2}-1, t\right) & \text { if } x_{2}-1>\bar{x}_{2}(t) \\
r_{2}+p_{1}-g & \text { if } x_{2}-1 \leq \bar{x}_{2}(t)<x_{2}\end{cases}
\end{gathered}
$$

Given that $\alpha\left(x_{2}, t\right)$ is non-increasing in $x_{2}$, it is clear from equation (12) that $\alpha\left(x_{2}, t-1\right)$ is nonincreasing in $x_{2}$ for all $x_{2} \geq 1$, finishing the induction proof.

\section{Proof of Proposition 2}

For the ease of representation, we drop the subscripts of $x_{2}$ and $\bar{x}_{2}(t)$ in the following proof.

Induction assumption: $\bar{x}(t) \geq \bar{x}(t+1)$ and $\alpha(x, t) \geq \alpha(x, t+1) \quad \forall x \in[1, \bar{x}(t)]$

We need to show $\bar{x}(t-1) \geq \bar{x}(t)$ and $\alpha(x, t-1) \geq \alpha(x, t) \quad \forall x \in[1, \bar{x}(t-1)]$.

From the definition of $\bar{x}(t)$ (page 13), $\delta \alpha(x, t)<r_{2}+p_{1}-g+h_{2}$ if $x>\bar{x}(t)$ and $\delta \alpha(x, t) \geq r_{2}+p_{1}-g+h_{2}$ if $x \leq \bar{x}(t)$. 
Assume for contradiction that $\bar{x}(t-1)<\bar{x}(t)$, we then have $\delta \alpha(\bar{x}(t-1), t) \geq r_{2}+p_{1}-g+h$.

From equation (12), we have:

$$
\begin{aligned}
& \alpha(\bar{x}(t), t-1)-\alpha(\bar{x}(t), t) \\
& =(1-\lambda) \delta[\alpha(\bar{x}(t), t)-\alpha(\bar{x}(t), t+1)]+\lambda_{2} \delta[\alpha(\bar{x}(t)-1, t)-\alpha(\bar{x}(t)-1, t+1)] \\
& +\lambda_{1}\left\{\begin{array}{lc}
\delta[\alpha(\bar{x}(t), t)-\alpha(\bar{x}(t), t+1)] & \text { if } \bar{x}(t) \leq \bar{x}(t+1) \\
\delta[\alpha(\bar{x}(t), t)-\alpha(\bar{x}(t)-1, t+1)] & \text { if } \bar{x}(t+1)<\bar{x}(t)-1 \\
\delta \alpha(\bar{x}(t), t)-\left(r_{2}+p_{1}-g+h_{2}\right) & \text { if } \bar{x}(t)-1 \leq \bar{x}(t+1)
\end{array}\right.
\end{aligned}
$$

By induction assumption, the terms $\alpha(\bar{x}(t), t)-\alpha(\bar{x}(t), t+1)$ and $\alpha(\bar{x}(t)-1, t)-\alpha(\bar{x}(t)-1, t+1)$ are nonnegative. We know from the definition of $\bar{x}(t)$ that $\delta \alpha(\bar{x}(t), t) \geq r_{2}+p_{1}-g+h$. Under the condition $\bar{x}(t+1)<\bar{x}(t)-1$, we have $\alpha(\bar{x}(t), t) \geq\left(r_{2}+p_{1}-g+h\right) / \delta>\alpha(\bar{x}(t)-1, t+1)$. Thus the term $\alpha(\bar{x}(t), t)-\alpha(\bar{x}(t)-1, t+1)$ is positive. Hence we obtain $\alpha(\bar{x}(t), t-1)>\alpha(\bar{x}(t), t) \geq r_{2}+p_{1}-g+h$ which implies that $\bar{x}(t-1)>\bar{x}(t)$. Therefore, we have a contradiction. Thus we have proved $\bar{x}(t-1) \geq \bar{x}(t)$.

Next we need to show $\alpha(x, t-1) \geq \alpha(x, t) \quad \forall x \in[1, \bar{x}(t-1)]$.

Given $\bar{x}(t-1) \geq \bar{x}(t) \quad \forall t=\tau+1, \tau+2, \ldots, \tau+T$, we can separate the interval $[1, \bar{x}(t-1)]$ into three subintervals : i) $x \in[1, \bar{x}(t+1)]$, ii) $x \in(\bar{x}(t+1), \bar{x}(t)]$, and iii) $x \in(\bar{x}(t), \bar{x}(t-1)]$.

i) $\forall x \in[1, \bar{x}(t+1)]$

$$
\begin{aligned}
& \alpha(x, t-1)-\alpha(x, t) \\
& =(1-\lambda) \delta[\alpha(x, t)-\alpha(x, t+1)]+\lambda_{2} \delta[\alpha(x-1, t)-\alpha(x-1, t+1)]+\lambda_{1} \delta[\alpha(x, t)-\alpha(x, t+1)]
\end{aligned}
$$

By induction assumption, the RHS terms are all nonnegative, thus

$$
\alpha(x, t-1) \geq \alpha(x, t) \quad \forall x \in[1, \bar{x}(t+1)]
$$

ii) $\forall x \in(\bar{x}(t+1), \bar{x}(t)]$, 


$$
\begin{gathered}
\alpha(x, t-1)-\alpha(x, t)=(1-\lambda) \delta[\alpha(x, t)-\alpha(x, t+1)]+\lambda_{2} \delta[\alpha(x-1, t)-\alpha(x-1, t+1)] \\
+\lambda_{1}\left\{\begin{array}{l}
\delta[\alpha(x, t)-\alpha(x-1, t+1)] \quad \text { if } x-1>\bar{x}(t+1) \\
\delta \alpha(x, t)-\left(r_{2}+p_{1}-g+h_{2}\right) \quad \text { if } x-1 \leq \bar{x}(t+1)<x
\end{array}\right.
\end{gathered}
$$

$\delta \alpha(x, t)-\left(r_{2}+p_{1}-g+h_{2}\right)$ is nonnegative as $x \leq \bar{x}(t)$,

$\alpha(x, t)-\alpha(x-1, t+1)$ is nonnegative because for $x \leq \bar{x}(t)$ and $x-1>\bar{x}(t+1)$ we have

$\alpha(x, t) \geq\left(r_{2}+p_{1}-g+h_{2}\right) / \delta$ and $\alpha(x-1, t+1) \leq\left(r_{2}+p_{1}-g+h_{2}\right) / \delta$

iii) $x \in(\bar{x}(t), \bar{x}(t-1)]$

since $x>\bar{x}(t)$ and $x \leq \bar{x}(t-1)$, condition (7) implies $\alpha(x, t) \leq\left(r_{2}+p_{1}-g+h_{2}\right) / \delta$ and $\alpha(x, t-1) \geq\left(r_{2}+p_{1}-g+h_{2}\right) / \delta$, thus $\alpha(x, t-1)-\alpha(x, t)$ is nonnegative.

Therefore, we have $\alpha(x, t-1) \geq \alpha(x, t) \quad \forall x \in[1, \bar{x}(t-1)]$, finishing the induction step.

Now we establish the base case. That is, we need to show $\bar{x}(\tau+T-1) \geq \bar{x}(\tau+T)$ and that $\alpha(x, \tau+T-1) \geq \alpha(x, \tau+T) \quad \forall x \in[1, \bar{x}(\tau+T-1)]$

From assumption (4), $\delta \alpha(x, \tau+T-1)<r_{2}+p_{1}-g+h_{2}$ for $x \geq 1$; thus it is optimal to substitute at period $\tau+T-1$ whenever inventory is positive, or equivalently $\bar{x}(\tau+T)=0$. Hence $\bar{x}(\tau+T-1) \geq \bar{x}(\tau+T)$ holds

Since $\alpha(x, \tau+T-1)=-h_{2}+\delta s_{2}$ for $x \geq 2$, we have $\delta \alpha(x, \tau+T-1)<\delta s_{2} \leq r_{2}+p_{1}-g+h_{2}$ for $x \geq 2$. Thus $\bar{x}(\tau+T-1)<2$.

If $\bar{x}(\tau+T-1)<1$, the set $[1, \bar{x}(\tau+T-1)]$ contains no integral points; if $\bar{x}(\tau+T-1) \geq 1$, $\alpha(1, \tau+T-1) \geq\left(r_{2}+p_{1}-g+h_{2}\right) / \delta>s_{2}=\alpha(1, \tau+T)$. Therefore the base case is true.

\section{Proof of Proposition 3}

For the ease of representation, we drop the subscripts of $x_{2}$ and $\bar{x}_{2}(t)$ in the following proof. 
When $h=0$ and $\delta=1$, equation (4-8) becomes:

$$
\begin{aligned}
& \alpha(x, t-1)=(1-\lambda(t)) \alpha(x, t)+\lambda_{2}(t) \alpha(x-1, t) \\
&+\lambda_{1}(t) \begin{cases}\alpha(x, t) & \text { if } x \leq \bar{x}(t) \\
\alpha(x-1, t) & \text { if } x-1>\bar{x}(t) \\
r_{2}+p_{1}-g & \text { if } x-1 \leq \bar{x}(t)<x\end{cases}
\end{aligned}
$$

Thus

$$
\begin{aligned}
& \alpha(x, t-1)-\alpha(x, t) \\
& = \begin{cases}\lambda_{2}(t)[\alpha(x-1, t)-\alpha(x, t)] & \text { if } x \leq \bar{x}(t) \\
\lambda(t)[\alpha(x-1, t)-\alpha(x, t)] & \text { if } x-1>\bar{x}(t) \\
\lambda_{2}(t)[\alpha(x-1, t)-\alpha(x, t)]+\lambda_{1}(t)\left[r_{2}+p_{1}-g-\alpha(x, t)\right] & \text { if } x-1 \leq \bar{x}(t)<x\end{cases}
\end{aligned}
$$

Since $\alpha(x, t)$ is non-increasing in $x, \alpha(x-1, t)-\alpha(x, t)$ is nonnegative, the term $r_{2}+p_{1}-g-\alpha(x, t)$ is nonnegative for $x>\bar{x}(t)$ (by the definition of $\bar{x}(t)$ on page 13). Therefore, we have $\alpha(x, t-1) \geq \alpha(x, t) \quad \forall \mathrm{t}=\tau+1, \tau+2, \ldots, \tau+\mathrm{T} . \square$

\section{Proof of Proposition 4}

For the ease of representation, we drop the subscripts of $x_{2}$ and $\bar{x}_{2}(t)$ in the proof.

In order to show that $\bar{x}(t)$ is a unimodal, it is sufficient to show the following:

a) $\bar{x}(t+1) \geq \bar{x}(t) \Rightarrow \bar{x}(t) \geq \bar{x}(t-1)$

b) $\bar{x}(t-1) \geq \bar{x}(t) \Rightarrow \bar{x}(t) \geq \bar{x}(t+1)$

We prove a) first.

Given $\bar{x}(t+1) \geq \bar{x}(t)$, we consider the following five cases:

i) $\bar{x}(t) \leq x-1<x \leq \bar{x}(t+1)$

ii) $\bar{x}(t) \leq \bar{x}(t+1) \leq x-1<x$

iii) $x-1<x \leq \bar{x}(t) \leq \bar{x}(t+1)$

iv) $\bar{x}(t) \leq x-1 \leq \bar{x}(t+1)<x$ 
v) $x-1 \leq \bar{x}(t) \leq x \leq \bar{x}(t+1)$

It is easy to show that for each case $\alpha(x, t-1)<\alpha(x, t)$, which in turn yields $\bar{x}(t) \geq \bar{x}(t-1)$

We then prove b) $\bar{x}(t-1) \geq \bar{x}(t) \Rightarrow \bar{x}(t) \geq \bar{x}(t+1)$.

Assume for contradiction that $\bar{x}(t) \leq \bar{x}(t+1)$, from a), we have $\bar{x}(t-1) \leq \bar{x}(t)$, which contradicts $\bar{x}(t-1) \geq \bar{x}(t)$.

From a) and b), it is easy to see that $\bar{x}(t)$ is unimodal in $t$.

\section{Proof of Proposition 5}

Define $\alpha\left(x_{1}, x_{2}, t\right) \equiv V\left(x_{1}, x_{2}, t+1\right)-V\left(x_{1}, x_{2}-1, t+1\right)$

and $\beta\left(x_{1}, x_{2}, t\right) \equiv V\left(x_{1}, x_{2}, t+1\right)-V\left(x_{1}-1, x_{2}, t+1\right)$.

For ease of exposition, we also define $\beta\left(0, x_{2}, t\right) \equiv\left(v_{1}+h_{2}\right) / \delta$

To show that $V$ is jointly concave, it is sufficient and necessary to show that $\alpha\left(x_{1}, x_{2}, t\right)$ and $\beta\left(x_{1}, x_{2}, t\right)$ are non-increasing in $x_{1}$ and $x_{2}$. We first derive the expressions for the partial and cross partial differences.

From equations (1),

For $x_{1} \geq 1, x_{2} \geq 1$

$$
\begin{gathered}
\alpha\left(x_{1}, x_{2}, t-1\right)=-h_{2}+(1-\lambda(t)) \delta \alpha\left(x_{1}, x_{2}, t\right)+\lambda_{2}(t) \delta \alpha\left(x_{1}, x_{2}-1, t\right) \\
+\lambda_{1}(t) \delta \alpha\left(x_{1}-1, x_{2}, t\right)
\end{gathered}
$$

For $x_{1}=0, x_{2} \geq 1$, from equation (12), we have

$$
\begin{array}{r}
\alpha\left(0, x_{2}, t-1\right)=-h_{2}+(1-\lambda(t)) \delta \alpha\left(0, x_{2}, t\right)+\lambda_{2}(t) \delta \alpha\left(0, x_{2}-1, t\right) \\
+\lambda_{1}(t) \begin{cases}\delta \alpha\left(0, x_{2}, t\right) & \text { if } x_{2} \leq \bar{x}(t) \\
\delta \alpha\left(0, x_{2}-1, t\right) & \text { if } x_{2}-1>\bar{x}(t) \\
r_{2}+p_{1}-g+h_{2} & \text { if } x_{2}-1 \leq \bar{x}(t)<x_{2}\end{cases}
\end{array}
$$


For $x_{1} \geq 2, x_{2} \geq 1$

$$
\begin{aligned}
\beta\left(x_{1}, x_{2}, t-1\right)=- & h_{1}+(1-\lambda(t)) \delta \beta\left(x_{1}, x_{2}, t\right)+\lambda_{2}(t) \delta \beta\left(x_{1}, x_{2}-1, t\right) \\
& +\lambda_{1}(t) \delta \beta\left(x_{1}-1, x_{2}, t\right)
\end{aligned}
$$

For $x_{1}=1, x_{2} \geq 1$

$$
\begin{aligned}
\beta\left(1, x_{2}, t-1\right)=-h_{1} & +(1-\lambda(t)) \delta \beta\left(1, x_{2}, t\right)+\lambda_{2}(t) \delta \beta\left(1, x_{2}-1, t\right) \\
& +\lambda_{1}(t)\left\{\begin{array}{l}
\left(v_{1}+h_{1}\right) \quad \text { if } x_{2}<\bar{x}(t) \\
\delta \alpha\left(0, x_{2}, t\right)+r_{1}-r_{2}-h_{2}+g+h_{1} \text { o.w. }
\end{array}\right.
\end{aligned}
$$

For $x_{1} \geq 1, x_{2}=0$

$\beta\left(x_{1}, 0, t-1\right)=-h_{1}+(1-\lambda(t)) \delta \beta\left(x_{1}, 0, t\right)+\lambda_{2}(t) \delta \beta\left(x_{1}, 0, t\right)+\lambda_{1}(t) \delta \beta\left(x_{1}-1,0, t\right)$

Therefore, we obtain the following recursive equations.

Regarding the partial difference $\alpha\left(x_{1}, x_{2}\right)-\alpha\left(x_{1}, x_{2}-1\right)$

For $x_{1} \geq 1, x_{2} \geq 2$

$$
\begin{gathered}
\alpha\left(x_{1}, x_{2}, t-1\right)-\alpha\left(x_{1}, x_{2}-1, t-1\right)=(1-\lambda(t)) \delta\left[\alpha\left(x_{1}, x_{2}, t\right)-\alpha\left(x_{1}, x_{2}-1, t\right)\right] \\
+\lambda_{2}(t) \delta\left[\alpha\left(x_{1}, x_{2}-1, t\right)-\alpha\left(x_{1}, x_{2}-2, t\right)\right] \\
+\lambda_{1}(t) \delta\left[\alpha\left(x_{1}-1, x_{2}, t\right)-\alpha\left(x_{1}-1, x_{2}-1, t\right)\right]
\end{gathered}
$$

For $x_{1}=0, x_{2} \geq 2$

$$
\begin{aligned}
& \alpha\left(0, x_{2}, t-1\right)-\alpha\left(0, x_{2}-1, t-1\right) \\
& =(1-\lambda(t)) \delta\left[\alpha\left(0, x_{2}, t\right)-\alpha\left(0, x_{2}-1, t\right)\right] \\
& +\lambda_{2}(t) \delta\left[\alpha\left(0, x_{2}-1, t\right)-\alpha\left(0, x_{2}-2, t\right)\right] \\
& +\lambda_{1}(t) \begin{cases}\delta\left[\alpha\left(0, x_{2}, t\right)-\alpha\left(0, x_{2}-1, t\right)\right] & \text { if } x_{2} \leq \bar{x}(t) \\
\delta\left[\alpha\left(0, x_{2}-1, t\right)-\alpha\left(0, x_{2}-2, t\right)\right] & \text { if } x_{2}-2>\bar{x}(t) \\
\delta \alpha\left(0, x_{2}-1, t\right)-\left(r_{2}+p_{1}-g+h_{2}\right) & \text { if } x_{2}-2 \leq \bar{x}(t)<x_{2}-1<x_{2} \\
\left(r_{2}+p_{1}-g+h_{2}\right)-\delta \alpha\left(0, x_{2}-1, t\right) & \text { if } x_{2}-2<x_{2}-1 \leq \bar{x}(t)<x_{2}\end{cases}
\end{aligned}
$$

From condition (7), $\delta \alpha\left(0, x_{2}-1, t\right)-\left(r_{2}+p_{1}-g+h_{2}\right) \leq 0$ if $\bar{x}(t)<x_{2}-1$ and $\left(r_{2}+p_{1}-g+h_{2}\right)-\delta \alpha\left(0, x_{2}-1, t\right) \leq 0$ if $x_{2}-1 \leq \bar{x}(t)$. 
Therefore, the last two items within the curly bracket are both non-positive.

Regarding the partial difference $\beta\left(x_{1}, x_{2}\right)-\beta\left(x_{1}-1, x_{2}\right)$ :

For $x_{1} \geq 3, x_{2} \geq 1$

$$
\begin{aligned}
\beta\left(x_{1}, x_{2}, t-1\right)-\beta\left(x_{1}-1,\right. & \left.x_{2}, t-1\right)=(1-\lambda(t)) \delta\left[\beta\left(x_{1}, x_{2}, t\right)-\beta\left(x_{1}-1, x_{2}, t\right)\right] \\
+ & \lambda_{2}(t) \delta\left[\beta\left(x_{1}, x_{2}-1, t\right)-\beta\left(x_{1}-1, x_{2}-1, t\right)\right] \\
& +\lambda_{1}(t) \delta\left[\beta\left(x_{1}-1, x_{2}, t\right)-\beta\left(x_{1}-2, x_{2}, t\right)\right]
\end{aligned}
$$

For $x_{1}=2, x_{2} \geq 1$

$$
\begin{aligned}
\beta\left(2, x_{2}, t-1\right)-\beta\left(1, x_{2}, t-1\right) & =(1-\lambda(t)) \delta\left[\beta\left(2, x_{2}, t\right)-\beta\left(1, x_{2}, t\right)\right] \\
+ & \lambda_{2}(t) \delta\left[\beta\left(2, x_{2}-1, t\right)-\beta\left(1, x_{2}-1, t\right)\right] \\
+ & \lambda_{1}(t)\left\{\begin{array}{l}
\delta \beta\left(1, x_{2}, t\right)-\left(v_{1}+h_{1}\right) \\
\delta\left[\beta\left(1, x_{2}, t\right)-\alpha\left(0, x_{2}, t\right)\right]+r_{2}+h_{2}-r_{1}-h_{1}-g \quad \text { if } x_{2}<\bar{x}(t)
\end{array}\right.
\end{aligned}
$$

Regarding the cross partial difference $\alpha\left(x_{1}, x_{2}\right)-\alpha\left(x_{1}-1, x_{2}\right)$ :

For $x_{1} \geq 2, x_{2} \geq 1$

$$
\begin{aligned}
\alpha\left(x_{1}, x_{2}, t-1\right)-\alpha\left(x_{1}-1,\right. & \left.x_{2}, t-1\right)=(1-\lambda(t)) \delta\left[\alpha\left(x_{1}, x_{2}, t\right)-\alpha\left(x_{1}-1, x_{2}, t\right)\right] \\
& +\lambda_{2}(t) \delta\left[\alpha\left(x_{1}, x_{2}-1, t\right)-\alpha\left(x_{1}-1, x_{2}-1, t\right)\right] \\
& +\lambda_{1}(t) \delta\left[\alpha\left(x_{1}-1, x_{2}, t\right)-\alpha\left(x_{1}-2, x_{2}, t\right)\right]
\end{aligned}
$$

For $x_{1}=1, x_{2} \geq 1$

$$
\begin{aligned}
\alpha\left(1, x_{2}, t-1\right)-\alpha\left(0, x_{2}, t-1\right) & =(1-\lambda(t)) \delta\left[\alpha\left(1, x_{2}, t\right)-\alpha\left(0, x_{2}, t\right)\right] \\
+ & \lambda_{2}(t) \delta\left[\alpha\left(1, x_{2}-1, t\right)-\alpha\left(0, x_{2}-1, t\right)\right] \\
+ & \lambda_{1}(t)\left\{\begin{array}{lr}
0 & \text { if } x_{2}<\bar{x}(t) \\
\delta\left[\alpha\left(0, x_{2}, t\right)-\alpha\left(0, x_{2}-1, t\right)\right] & \text { if } x_{2}-1>\bar{x}(t) \\
\delta \alpha\left(0, x_{2}, t\right)-\left(r_{2}+p_{1}-g+h_{2}\right) & \text { if } x_{2}-1 \leq \bar{x}(t)<x_{2}
\end{array}\right.
\end{aligned}
$$

From condition (7), $\delta \alpha\left(0, x_{2}, t\right)-\left(r_{2}+p_{1}-g+h_{2}\right)$ if $\bar{x}(t)<x_{2}$. Therefore, the last item within the curly bracket is non-positive.

Regarding the cross partial difference $\beta\left(x_{1}, x_{2}\right)-\beta\left(x_{1}, x_{2}-1\right)$ : 
For $x_{1} \geq 2, x_{2} \geq 2$

$$
\begin{aligned}
\beta\left(x_{1}, x_{2}, t-1\right)-\beta\left(x_{1}, x_{2}-\right. & 1, t-1)=(1-\lambda(t)) \delta\left[\beta\left(x_{1}, x_{2}, t\right)-\beta\left(x_{1}, x_{2}-1, t\right)\right] \\
& +\lambda_{2}(t) \delta\left[\beta\left(x_{1}, x_{2}-1, t\right)-\beta\left(x_{1}, x_{2}-2, t\right)\right] \\
& +\lambda_{1}(t) \delta\left[\beta\left(x_{1}-1, x_{2}, t\right)-\beta\left(x_{1}-1, x_{2}-1, t\right)\right]
\end{aligned}
$$

For $x_{1}=1, x_{2} \geq 2$

$$
\begin{aligned}
& \beta\left(1, x_{2}, t-1\right)-\beta\left(1, x_{2}-1, t-1\right)=(1-\lambda(t)) \delta\left[\beta\left(1, x_{2}, t\right)-\beta\left(1, x_{2}-1, t\right)\right] \\
& +\lambda_{2}(t) \delta\left[\beta\left(1, x_{2}-1, t\right)-\beta\left(1, x_{2}-2, t\right)\right] \\
& +\lambda_{1}(t)\left\{\begin{array}{l}
0 \quad \text { if } x_{2}<\bar{x}(t) \\
\delta \alpha\left(o, x_{2}, t\right)-\left(r_{2}+p_{1}-g+h_{2}\right) \text { if } x_{2}-1<\bar{x}(t)<x_{2} \\
\delta\left[\alpha\left(o, x_{2}, t\right)-\alpha\left(o, x_{2}-1, t\right)\right] \text { if } x_{2}-1>\bar{x}(t)
\end{array}\right.
\end{aligned}
$$

From condition (7), $\delta \alpha\left(0, x_{2}, t\right)-\left(r_{2}+p_{1}-g+h_{2}\right) \leq 0$ if $\bar{x}(t)<x_{2}$. Therefore, the second item within the curly bracket is non-positive.

For $x_{1} \geq 1, x_{2}=1$

$$
\begin{aligned}
\beta\left(x_{1}, 1, t-1\right)-\beta\left(x_{1}, 0, t-1\right) & =(1-\lambda(t)) \delta\left[\beta\left(x_{1}, 1, t\right)-\beta\left(x_{1}, 0, t\right)\right] \\
+ & \lambda_{1}(t) \delta\left[\beta\left(x_{1}-1,1, t\right)-\beta\left(x_{1}-1,0, t\right)\right]
\end{aligned}
$$

Next we show by induction that these partial and cross partial differences are non-positive.

We first establish the base case:

Since $V\left(x_{1}, x_{2}, \tau+T+1\right)=s_{1} x_{1}+s_{2} x_{2}$, it is easy to see that $\alpha\left(x_{1}, x_{2}, \tau+T\right)=s_{2}$ and

$$
\beta\left(x_{1}, x_{2}, \tau+T\right)=s_{1}
$$

Thus $\alpha\left(x_{1}, x_{2}, \tau+T\right)-\alpha\left(x_{1}, x_{2}-1, \tau+T\right)=0$

$$
\begin{aligned}
& \beta\left(x_{1}, x_{2}, \tau+T\right)-\beta\left(x_{1}-1, x_{2}, \tau+T\right)=0 \\
& \alpha\left(x_{1}, x_{2}, \tau+T\right)-\alpha\left(x_{1}-1, x_{2}, \tau+T\right)=0 \\
& \beta\left(x_{1}, x_{2}, \tau+T\right)-\beta\left(x_{1}, x_{2}-1, \tau+T\right)=0
\end{aligned}
$$

In addition, we have the following:

i) $\delta \beta\left(1, x_{2}, \tau+T\right)-\left(v_{1}+h_{1}\right) \leq 0$ 
ii) $\delta\left[\beta\left(1, x_{2}, \tau+T\right)-\alpha\left(0, x_{2}, \tau+T\right)\right]+r_{2}+h_{2}-r_{1}-h_{1}-g \leq 0$

Note that i) and ii) are direct results of assumptions made in equations (2) and (5).

Induction assumption:

i) $\delta \beta\left(1, x_{2}, t\right)-\left(v_{1}+h_{1}\right) \leq 0$

ii) $\delta\left[\beta\left(1, x_{2}, t\right)-\alpha\left(0, x_{2}, t\right)\right]+r_{2}+h_{2}-r_{1}-h_{1}-g \leq 0$

iii) $\alpha\left(x_{1}, x_{2}, t\right)-\alpha\left(x_{1}, x_{2}-1, t\right) \leq 0$

iv) $\alpha\left(x_{1}, x_{2}, t\right)-\alpha\left(x_{1}-1, x_{2}, t\right) \leq 0$

v) $\beta\left(x_{1}, x_{2}, t\right)-\beta\left(x_{1}-1, x_{2}, t\right) \leq 0$

vi) $\beta\left(x_{1}, x_{2}, t\right)-\beta\left(x_{1}, x_{2}-1, t\right) \leq 0$

Induction step:

i)

$$
\begin{aligned}
& \beta\left(1, x_{2}, t-1\right)=-h_{1}+(1-\lambda(t)) \delta \beta\left(1, x_{2}, t\right)+\lambda_{2}(t) \delta \beta\left(1, x_{2}-1, t\right) \\
& +\lambda_{1}(t)\left\{\begin{array}{l}
\left(v_{1}+h_{1}\right) \quad \text { if } x_{2}<\bar{x}(t) \\
\delta \alpha\left(0, x_{2}, t\right)+r_{1}-r_{2}-h_{2}+g+h_{1} \text { o.w. }
\end{array}\right. \\
& \leq-h_{1}+(1-\lambda(t)) \delta\left(v_{1}+h_{1}\right)+\lambda_{2}(t) \delta\left(v_{1}+h_{1}\right)
\end{aligned}
$$

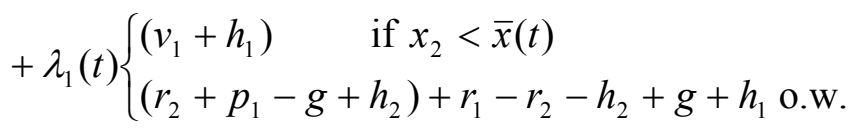

$$
\begin{aligned}
& =v_{1}
\end{aligned}
$$

(since $\left.x_{2}>\bar{x}(t) \Leftrightarrow \delta \alpha\left(0, x_{2}, t\right)<r_{2}+p_{1}-g+h_{2}\right)$

Thus $\delta \beta\left(1, x_{2}, t-1\right) \leq v_{1}+h_{1}$

ii)

$$
\begin{aligned}
& \beta\left(1, x_{2}, t-1\right)-\alpha\left(0, x_{2}, t-1\right) \\
& =-h_{1}+h_{2}+(1-\lambda(t)) \delta\left[\beta\left(1, x_{2}, t\right)-\alpha\left(0, x_{2}, t\right)\right]+\lambda_{2}(t) \delta\left[\beta\left(1, x_{2}-1, t\right)-\alpha\left(0, x_{2}-1, t\right)\right] \\
& +\lambda_{1}(t)\left\{\begin{array}{l}
v_{1}+h_{1}-\delta \alpha\left(0, x_{2}, t\right) \quad \text { if } x_{2}<\bar{x}(t) \\
r_{1}-r_{2}-h_{2}+h_{1}+g+\delta\left[\alpha\left(0, x_{2}, t\right)-\alpha\left(0, x_{2}-1, t\right)\right] \text { if } x_{2}-1>\bar{x}(t) \\
r_{1}-r_{2}-h_{2}+h_{1}+g+\delta \alpha\left(0, x_{2}, t\right)-\left(r_{2}+p_{1}-g+h_{2}\right) \text { o.w. }
\end{array}\right.
\end{aligned}
$$




$$
\begin{aligned}
& \leq-h_{1}+h_{2}+(1-\lambda(t))\left(r_{1}+h_{1}-r_{2}-h_{2}+g\right)+\lambda_{2}(t)\left(r_{1}+h_{1}-r_{2}-h_{2}+g\right) \\
& +\lambda_{1}(t) \begin{cases}v_{1}+h_{1}-\left(r_{2}+p_{1}-g+h_{2}\right) & \text { if } x_{2}<\bar{x}(t) \\
r_{1}-r_{2}-h_{2}+h_{1}+g & \text { if } x_{2}-1>\bar{x}(t) \\
r_{1}-r_{2}-h_{2}+h_{1}+g & \text { o.w. }\end{cases} \\
& \leq-h_{1}+h_{2}+(1-\lambda(t))\left(r_{1}-r_{2}-h_{2}+h_{1}+g\right)+\lambda_{2}(t)\left(r_{1}-r_{2}-h_{2}+h_{1}+g\right)+\lambda_{1}(t)\left(r_{1}-r_{2}-h_{2}+h_{1}+g\right) \\
& =r_{1}-r_{2}+g
\end{aligned}
$$

Thus $\delta\left[\beta\left(1, x_{2}, t-1\right)-\alpha\left(0, x_{2}, t-1\right)\right] \leq \delta\left(r_{1}-r_{2}+g\right)$

From assumption (6), $r_{1}-\left(r_{2}-g\right) \geq \frac{h_{2}-h_{1}}{1-\delta}$, equivalently,

$\left[r_{1}+h_{1}-\left(r_{2}-g\right)-h_{2}\right] \geq \delta\left[r_{1}-\left(r_{2}+g\right)\right]$

Therefore, we have $\delta\left[\beta\left(1, x_{2}, t-1\right)-\alpha\left(0, x_{2}, t-1\right)\right] \leq r_{1}-r_{2}+g-h_{2}+h_{1}$

Inductions for iii)-vi) are straightforward from the recursion equations.

Therefore, we have shown that $\alpha\left(x_{1}, x_{2}, t\right)$ and $\beta\left(x_{1}, x_{2}, t\right)$ are non-increasing in $x_{1}$ and $x_{2}$.

\section{Proof of Proposition 7 (Order-up-to Policy)}

We can rewrite equation (11) as

$$
N W\left(x_{1}, x_{2}, x_{1}^{0}\right)=c_{1} x_{1}^{0}+N W\left(x_{1}, x_{2}\right) \quad \forall x_{1} \geq x_{1}^{0}
$$

where $N W\left(x_{1}, x_{2}\right)$ is as defined in equation (10).

Let $\left(x_{1}^{*}, x_{2}^{*}\right)$ be the optimal solution that maximizes $N W\left(x_{1}, x_{2}\right)$.

If $x_{1}^{*} \geq x_{1}^{0}$, then $\left(x_{1}^{*}, x_{2}^{*}\right)$ also maximizes $N W\left(x_{1}, x_{2}, x_{1}^{0}\right)$.

If $x_{1}^{*}<x_{1}^{0}$, then $\left(x_{1}^{0}, x_{2}\left(x_{1}^{0}\right)\right)$ maximizes $N W\left(x_{1}, x_{2}, x_{1}^{0}\right)$ due to the concavity of $N W$, where $x_{2}\left(x_{1}^{0}\right)$ is the optimal quantity that maximizes $N W\left(x_{1}, x_{2}\right)$ given $x_{1}=x_{1}^{0}$

Therefore, the optimal order quantities are $\left(x_{1}^{*}-x_{1}^{0}, x_{2}^{*}\right)$ if $x_{1}^{0}<x_{1}^{*}$ and $\left(0, x_{2}\left(x_{1}^{0}\right)\right)$ if $x_{1}^{0} \geq x_{1}^{*}$.

Next we show that if $x_{1}^{0} \geq x_{1}^{*}$ then $x_{2}\left(x_{1}^{0}\right) \leq x_{2}^{*}$. 
This follows directly from the joint concavity of $N W . x_{2}\left(x_{1}^{0}\right)$ is the optimal quantity given $x_{1}^{0}$, thus $\left.\frac{\partial N W}{\partial x_{2}}\right|_{\left(x_{0}, x_{2}\left(x_{1}^{0}\right)\right)}=0$. Since $x_{1}^{0} \geq x_{1}^{*}$ and $\frac{\partial^{2} N W}{\partial x_{2} \partial x_{1}} \leq 0$, we have $\left.\frac{\partial N W}{\partial x_{2}}\right|_{\left(x_{1}^{*}, x_{2}\left(x_{1}^{0}\right)\right)} \leq 0$.

$N W\left(x_{1}, x_{2}\right)$ is maximized at $\left(x_{1}^{*}, x_{2}^{*}\right)$, thus $\left.\frac{\partial N W}{\partial x_{2}}\right|_{\left(x_{1}^{*}, x_{2}^{*}\right)}=0$. Therefore, $\left.\frac{\partial N W}{\partial x_{2}}\right|_{\left(x_{1}^{*}, x_{2}\left(x_{1}^{0}\right)\right)} \leq\left.\frac{\partial N W}{\partial x_{2}}\right|_{\left(x_{1}^{*}, x_{2}^{*}\right)}$.

Since $\frac{\partial^{2} N W}{\partial x_{2}^{2}} \leq 0$, this implies $x_{2}\left(x_{1}^{0}\right) \leq x_{2}^{*}$ 